\title{
CONTRÔLABILITÉ EXACTE D'UN PROBLÈME AVEC CONDITIONS DE VENTCEL ÉVOLUTIVES POUR LE SYSTÈME LINÉAIRE DE L'ÉLASTICITÉ*
}

\author{
Amar HEMINNA
}

\begin{abstract}
In this work, we examine the exact controllability of the solution of a linear elasticity system, with evolutive Ventcel's conditions, (see:[3]), in a bounded domain of $\mathbb{R}^{3}$. We use the Hilbert uniqueness methode,(H.U.M), of J.L.LIONS, (see:[9]); some multipliers are defined on the boundary; the curvature tensor (see:[6]), appears when computing some boundary integrals. This work can be inserted in the framework of the study of the exact controllability and stabilisation of various problems with Ventcel's conditions (see:[3],[4]).
\end{abstract}

\section{Résumé}

On considère le problème de la contrôlabilité exacte de la solution du système linéaire de l'élasticité, avec des conditions de Ventcel évolutives, ( $c f .[3])$, dans un domaine borné de $\mathbb{R}^{3}$. L'obtention d'une relation, en vue de la mise en oeuvre de la méthode d'unicité hilbertienne,(H.U.M.), de J.L. Lions, (cf.[9]), a donné lieu à l'utilisation de multiplicateurs définis sur le bord du domaine; le tenseur de courbure de la frontière, $(c f .[6])$, apparait lors du calcul de certaines intégrales de bord. Ce travail entre dans le cadre de l'étude de la contrôlabilité exacte et de la stabilisation de divers problèmes de Ventcel, (cf.[3],[4]). \footnotetext{
Lyon.

${ }^{*}$ Ce travail a été en partie réalisé lors d'un séjour au D.M.I. de l'École Centrale de
}

2000 Mathematics Subject Classification: 93B03, 93B05, 93D15.

Servicio de Publicaciones. Universidad Complutense. Madrid, 2001 


\section{Introduction}

Dans ce travail on étudie la contrôlabilité exacte de la solution du système linéaire de l'élasticité, avec des conditions de Ventcel évolutives. Les problèmes de Ventcel sont caractérisés par la présence d'opérateurs différentiels tangentiels de même ordre que l'opérateur principal.

Soit $\Omega$ un ouvert borné de $\mathbb{R}^{3}$ de frontière $\Gamma$ de classe $C^{3}$ (pour donner un sens au tenseur de courbure $c f .[6])$. On note $\nu$ la normale unitaire sortante. Soit $\mathbf{u}=\left(u_{1}, u_{2}, u_{3}\right)$ un champ vectoriel régulier défini dans $\Omega$; on pose $\epsilon_{i j}(\mathbf{u})=\frac{1}{2}\left(u_{\imath, \jmath}+u_{\jmath, \imath}\right)$ et $\sigma(\mathbf{u})=2 \mu \epsilon(\mathbf{u})+\lambda(\operatorname{div}(\mathbf{u})) I_{3}$, où $\lambda$ et $\mu$ sont les coefficents de Lamé supposés constants, $I_{3}$ est la matrice identité de $\mathbb{R}^{3}$ et $u_{\imath, \jmath}=\partial_{\jmath} u_{\imath}$. Soit $m \in \Gamma$; on désigne par $T_{m}(\Gamma)$ le plan tangent en $m$ à $\Gamma, \pi(m)$ la projection orthogonale sur $T_{m}(\Gamma)$. Soit $\mathbf{v} \in C^{1}\left(\bar{\Omega}, \mathbb{R}^{3}\right)$; on pose: $\mathbf{v}(m)=\mathbf{v}_{T}(m)+v_{\nu}(m) . \nu(m)$ où $\mathbf{v}_{T}(m)=\pi(m) \mathbf{v}(m)$ est la projection orthogonale de $\mathbf{v}(m)$ sur $T_{m}(\Gamma)$. On note $\partial_{m}$ et $\partial_{\nu}$ les dérivations tangentielle et normale, $\left(\partial_{m} \nu\right)$ l'opérateur de courbure sur $\Gamma$ et $\pi \partial_{m} \mathbf{v}_{T} \pi$ la dérivée covariante du champ $\mathbf{v}_{T} ; \partial_{m} \nu$ et $\pi \partial_{m} \mathbf{v}_{T} \pi$ sont deux champs d'endomorphismes du plan tangent. On a alors sur $\Gamma$, (cf. [1],[7],[12]):

$$
\epsilon(\mathbf{v})=\epsilon_{T}(\mathbf{v})+\nu \overline{\epsilon_{S}}(\mathbf{v})+\epsilon_{S}(\mathbf{v}) \bar{\nu}+\epsilon_{\nu}(\mathbf{v}) \nu \bar{\nu}
$$

avec

$$
\begin{aligned}
& 2 \epsilon_{T}(\mathbf{v})=\pi \partial_{m} \mathbf{v}_{T} \pi+\overline{\pi \partial_{m} \mathbf{v}_{T} \pi}+2 v_{\nu} \partial_{m} \nu \\
& 2 \epsilon_{S}(\mathbf{v})=\partial_{\nu} \mathbf{v}_{T}+\overline{\partial_{m} v_{\nu}}-\left(\partial_{m} \nu\right) \mathbf{v}_{T}, \quad \epsilon_{\nu}(\mathbf{v})=\partial_{\nu} v_{\nu}
\end{aligned}
$$

et

$$
\sigma(\mathbf{v})=\sigma_{T}(\mathbf{v})+\nu \overline{\sigma_{S}}(\mathbf{v})+\sigma_{S}(\mathbf{v}) \bar{\nu}+\sigma_{\nu}(\mathbf{v}) \nu \bar{\nu}
$$

avec

$$
\begin{gathered}
\sigma_{T}(\mathbf{v})=2 \mu \epsilon_{T}(\mathbf{v})+\lambda\left(\operatorname{tr}\left(\epsilon_{T}(\mathbf{v})\right)+\epsilon_{\nu}(\mathbf{v})\right) I_{2}, \quad \sigma_{S}(\mathbf{v})=2 \mu \epsilon_{S}(\mathbf{v}) \\
\sigma_{\nu}(\mathbf{v})=2 \mu \epsilon_{\nu}(\mathbf{v})+\lambda\left(\operatorname{tr}\left(\epsilon_{T}(\mathbf{v})\right)+\epsilon_{\nu}(\mathbf{v})\right)
\end{gathered}
$$

où la barre désigne le transposé d'un vecteur, d'un endomorphisme etc..., $I_{2}$ est la matrice identité du plan tangent et "tr" symbolise la trace d'une matrice. On pose:

$$
\begin{aligned}
& \sigma_{T}^{0}(\mathbf{v})=2 \mu \epsilon_{T}^{0}(\mathbf{v})+\lambda^{*} \operatorname{tr}\left(\epsilon_{T}^{0}(\mathbf{v})\right) I_{2} \\
& \operatorname{avec} \lambda^{*}=(2 \lambda \mu)(\lambda+2 \mu)^{-1} \text { et } \epsilon_{T}^{0}(\mathbf{v})=\epsilon_{T}(\mathbf{v})
\end{aligned}
$$


Soit $\tau_{T}$ un champ régulier d'endomorphismes du plan tangent à $\Gamma$; la divergence tangentielle de $\tau_{T}$ est le champ de formes linéaires sur le plan tangent noté $\operatorname{div}_{T} \tau_{T}$ défini par:

$$
\int_{\Gamma} \operatorname{div}_{T} \tau_{T} v_{T} d \Gamma=-\int_{\Gamma}\left(\tau_{T}: \pi \partial_{m} v_{T} \pi\right) d \Gamma, \quad \forall v_{T} \in \mathcal{D}(\Gamma, T(\Gamma))
$$

où $\left(\tau_{T}: \pi \partial_{m} v_{T} \pi\right)=\operatorname{tr}\left(\tau_{T} \cdot \pi \partial_{m} v_{T} \pi\right)$ et $\mathcal{D}(\Gamma, T(\Gamma))$ désigne l'espace des champs tangents à $\Gamma$ dont les composantes dans une base $\left\{a_{1}, a_{2}\right\}$ de $T_{m}(\Gamma)$ sont dans $\mathcal{D}(\Gamma)$. D'après $(1.3)$ on a: $\sigma(\mathbf{u}) . \nu=\sigma_{S}(\mathbf{u})+\sigma_{\nu}(\mathbf{u}) \nu$. On considère le système:

$$
\left\{\begin{array}{l}
\mathbf{u}^{\prime \prime}-\operatorname{div} \sigma(\mathbf{u})=\mathbf{f} \quad \text { dans } \quad Q_{T}=\Omega \times(0, T), \\
\mathbf{u}_{T}^{\prime \prime}+\sigma_{S}(\mathbf{u})-\overline{\operatorname{div}_{T} \sigma_{T}^{0}(\mathbf{u})=\mathbf{g}_{T}} \quad \text { sur } \quad \Sigma_{T}=\Gamma \times(0, T) \\
u_{\nu}^{\prime \prime}+\sigma_{\nu}(\mathbf{u})+\sigma_{T}^{0}(\mathbf{u}): \partial_{m} \nu=g_{\nu} \quad \text { sur } \quad \Sigma_{T} \\
\mathbf{u}(0)=\mathbf{u}_{1}^{0}, \quad \mathbf{u}^{\prime}(0)=\mathbf{u}_{1}^{1} \text { dans } \quad \Omega \\
\mathbf{u}_{\mid \Gamma}(0)=\mathbf{u}_{2}^{0}, \quad\left(\mathbf{u}_{\mid \Gamma}\right)^{\prime}(0)=\mathbf{u}_{2}^{1} \quad \text { sur }
\end{array}\right.
$$

où $\left({ }^{\prime}=\partial_{t}\right), \operatorname{div} \sigma(\mathbf{u})$ est le champ vectoriel de composantes $\sigma_{i j, j}(\mathbf{u}), i=$ $1,2,3, \operatorname{div}_{T} \sigma_{T}^{0}(\mathbf{u})$ est la divergence tangentielle du champ d'endomorphismes du plan tangent $\sigma_{T}^{0}(\mathbf{u})$ et $\left(\sigma_{T}^{0}(\mathbf{u}): \partial_{m} \nu\right)=\operatorname{tr}\left(\sigma_{T}^{0}(\mathbf{u}) \cdot \partial_{m} \nu\right)$.

Les conditions au bord dans (1.5) sont les conditions de Ventcel évolutives; elles sont obtenues par passage à la limite dans un problème de transmission posé dans l'ouvert formé de la jonction de l'ouvert $\Omega$ et d'une coque mince $\Omega_{-}^{\varepsilon}$ d'épaisseur $\varepsilon$ posée sur son bord, infiniment rigide et infiniment dense, lorsque l'épaisseur $\varepsilon$ de la coque tend vers 0 ; elles modélisent l'effet asymptotique du raidisseur $\Omega_{-}^{\varepsilon}$ (cf.[7] pour l'obtention de diverses conditions de Ventcel stationnaires).

On pose: $\mathbf{g}(m)=\mathbf{g}_{T}(m)+g_{\nu}(m) \nu(m)$ et on prend $\mathbf{f}=0$ dans $(1,5)$; il s'agit de trouver, pour des conditions initiales et un temps $\mathrm{T}$ convenables, un contrôle $\mathbf{g}$ tel que la solution $\mathbf{u}$ vérifie: $\mathbf{u}(T)=\mathbf{u}^{\prime}(T)=0$.

On considère les espaces:

$\star L^{2}(\Gamma, T(\Gamma)),\left(\right.$ resp. $\left.H^{1}(\Gamma, T(\Gamma))\right)$, l'espace des champs tangents $\mathbf{v}_{T}$ dont les composantes dans une base du plan tangent sont dans $L^{2}(\Gamma),\left(\operatorname{resp} . H^{1}(\Gamma)\right)$.

$\star \mathcal{H}=\mathbb{L}^{2}(\Omega) \times L^{2}(\Gamma, T(\Gamma)) \times L^{2}(\Gamma)$

muni de la norme produit. 
$\star \mathbb{V}=\left\{\mathbf{v} \in \mathbb{H}^{1}(\Omega): \mathbf{v}_{T \mid \Gamma} \in H^{1}(\Gamma, T(\Gamma))\right\}$

muni de la norme:

$$
\|\mathbf{v}\|_{\mathbb{V}}=\left(\|\mathbf{v}\|_{\mathbb{H}^{1}(\Omega)}^{2}+\left\|\mathbf{v}_{T}\right\|_{H^{1}(\Gamma, T(\Gamma))}^{2}\right)^{\frac{1}{2}}
$$

Nous verrons dans la proposition 2.4 que cette norme est équivalente à la norme $\|\cdot\|$ définie par:

$$
\|\mathbf{v}\|=\left(\int_{\Omega} \sigma(\mathbf{v}): \epsilon(\mathbf{v}) d x+\int_{\Gamma}\left(\sigma_{T}^{0}(\mathbf{v}): \epsilon_{T}^{0}(\mathbf{v})+|\mathbf{v}|^{2}\right) d \Gamma\right)^{\frac{1}{2}}
$$

et l'espace:

$\star \mathcal{V}=\left\{\left(\mathbf{v}_{1}, \mathbf{v}_{2}\right): \mathbf{v}_{1} \in \mathbb{V}, \mathbf{v}_{2}=\mathbf{v}_{1 \mid \Gamma}\right\}$

muni de la norme:

$$
\left\|\left(\mathbf{v}_{1}, \mathbf{v}_{2}\right)\right\|_{\mathcal{V}}=\left\|\mathbf{v}_{1}\right\|_{\mathbb{V}}
$$

On considère sur $\mathcal{H}$ l'opérateur non borné :

$$
\mathcal{A}=\left(\begin{array}{cc}
-\operatorname{div} \sigma(.) & 0 \\
\sigma_{S}(.) & -\operatorname{div}_{T} \sigma_{T}^{0}(.) \\
\sigma_{\nu}(.) & \sigma_{T}^{0}(.): \partial_{m} \nu
\end{array}\right)
$$

de domaine $D(\mathcal{A})=\{U \in \mathcal{V}: \mathcal{A} U \in \mathcal{H}\}$. La norme de $D(\mathcal{A})$ est définie par:

$$
\begin{gathered}
\left\|\left(\mathbf{u}_{1}, \mathbf{u}_{2}\right)\right\|_{D(\mathcal{A})}=\left(\left\|\mathbf{u}_{1}\right\|_{\mathbb{V}}^{2}+\left\|\operatorname{div} \sigma\left(\mathbf{u}_{1}\right)\right\|_{\mathbb{L}^{2}(\Omega)}^{2}+\right. \\
\left.\left\|\sigma_{S}\left(\mathbf{u}_{1}\right)-{\overline{\operatorname{div}} \sigma_{T}^{0}}_{T}^{0}\left(\mathbf{u}_{2}\right)\right\|_{L^{2}(\Gamma, T(\Gamma))}^{2}+\left\|\sigma_{\nu}\left(\mathbf{u}_{1}\right)+\sigma_{T}^{0}\left(\mathbf{u}_{2}\right): \partial_{m} \nu\right\|_{L^{2}(\Gamma)}^{2}\right)^{\frac{1}{2}}
\end{gathered}
$$

On montre le résultat d'existence et d'unicité suivant:

Theoreme 1.1. (i) Si $\mathbf{f} \in L^{1}\left(0, T ; \mathbb{L}^{2}(\Omega)\right)$, $\mathbf{g} \in L^{1}\left(0, T ; \mathbb{L}^{2}(\Gamma)\right),\left(\mathbf{u}_{1}^{0}, \mathbf{u}_{2}^{0}\right)$ $\in \mathcal{V}$ et $\left(\mathbf{u}_{1}^{1}, \mathbf{u}_{2}^{1}\right) \in \mathcal{H}$ alors le problème (1.5) admet une solution (faible) unique $\mathbf{u}$ telle que:

$$
\left(\mathbf{u}, \mathbf{u}_{\mid \Gamma}\right) \in C^{0}([0, T] ; \mathcal{V}) \cap C^{1}([0, T] ; \mathcal{H})
$$


et il existe une constante positive $C_{1}$ ne dépendant que de $\Omega$ telle que:

$$
\begin{gathered}
\left\|\left(\mathbf{u}, \mathbf{u}_{\mid \Gamma}\right)\right\|_{L^{\infty}(0, T ; \mathcal{V})}+\left\|\left(\mathbf{u}^{\prime}, \mathbf{u}_{\mid \Gamma}^{\prime}\right)\right\|_{L^{\infty}(0, T ; \mathcal{H})} \leq C_{1}\left(\|\mathbf{f}\|_{L^{1}\left(0, T ; \mathbb{L}^{2}(\Omega)\right)}+\right. \\
\left.\|\mathbf{g}\|_{L^{1}\left(0, T ; \mathbb{L}^{2}(\Gamma)\right)}+\left\|\left(\mathbf{u}_{1}^{0}, \mathbf{u}_{2}^{0}\right)\right\|_{\mathcal{V}}+\left\|\left(\mathbf{u}_{1}^{1}, \mathbf{u}_{2}^{1}\right)\right\|_{\mathcal{H}}\right)
\end{gathered}
$$

(ii)Si $\mathbf{f} \in L^{1}(0, T ; \mathbb{V}), \mathbf{g}=\mathbf{f}_{\mid \Gamma},\left(\mathbf{u}_{1}^{0}, \mathbf{u}_{2}^{0}\right) \in D(\mathcal{A})$ et $\left(\mathbf{u}_{1}^{1}, \mathbf{u}_{2}^{1}\right) \in \mathcal{V}$ alors la solution $\mathbf{u}$ du problème (1.5) est telle que:

$$
\left(\mathbf{u}, \mathbf{u}_{\mid \Gamma}\right) \in C^{0}([0, T] ; D(\mathcal{A})) \cap C^{1}([0, T] ; \mathcal{V})
$$

et il existe une constante positive $C_{2}$ ne dépendant que de $\Omega$ telle que:

$$
\begin{gathered}
\left\|\left(\mathbf{u}, \mathbf{u}_{\mid \Gamma}\right)\right\|_{L^{\infty}(0, T ; D(\mathcal{A}))}+\left\|\left(\mathbf{u}^{\prime}, \mathbf{u}_{\mid \Gamma}^{\prime}\right)\right\|_{L^{\infty}(0, T ; \mathcal{V})} \leq C_{2}\left(\|\mathbf{f}\|_{L^{1}(0, T ; \mathbb{V})}+\right. \\
\left.+\left\|\left(\mathbf{u}_{1}^{0}, \mathbf{u}_{2}^{0}\right)\right\|_{D(\mathcal{A})}+\left\|\left(\mathbf{u}_{1}^{1}, \mathbf{u}_{2}^{1}\right)\right\|_{\mathcal{V}}\right)
\end{gathered}
$$

De plus on a:

$$
\left(\mathbf{u}, \mathbf{u}_{T}, \sigma(\mathbf{u}) . \nu\right) \in C^{0}\left([0, T] ; \mathbb{H}^{\frac{3}{2}}(\Omega) \times H^{2}(\Gamma, T(\Gamma)) \times \mathbb{L}^{2}(\Gamma)\right)
$$

et il existe une constante positive $C_{3}$ ne dépendant que de $\Omega$ telle que:

$$
\begin{aligned}
& \|\mathbf{u}\|_{L^{\infty}\left(0, T ; \mathbb{H}^{\frac{3}{2}}(\Omega)\right)}+\left\|\mathbf{u}_{T}\right\|_{L^{\infty}\left(0, T ; H^{2}(\Gamma, T(\Gamma))\right.}+ \\
& \| \sigma(\mathbf{u}) \cdot \nu) \|_{L^{\infty}\left(0, T ; \mathbb{L}^{2}(\Gamma, T(\Gamma))\right.} \leq \\
& \quad C_{3}\left(\|\mathbf{f}\|_{L^{1}(0, T ; \mathbb{V})}+\left\|\left(\mathbf{u}_{1}^{0}, \mathbf{u}_{2}^{0}\right)\right\|_{D(\mathcal{A})}+\left\|\left(\mathbf{u}_{1}^{1}, \mathbf{u}_{2}^{1}\right)\right\|_{\mathcal{V}}\right)
\end{aligned}
$$

On considère maintenant le problème:

$$
\left\{\begin{array}{l}
\varphi_{1}^{\prime \prime}-\operatorname{div} \sigma\left(\varphi_{1}\right)=f_{1} \text { dans } Q_{T} \\
\varphi_{2 T}^{\prime \prime}+\sigma_{S}\left(\varphi_{1}\right)-\operatorname{div}_{T} \sigma_{T}^{0}\left(\varphi_{2}\right)=f_{2 T} \quad \text { sur } \Sigma_{T} \\
\varphi_{2 \nu}^{\prime \prime}+\sigma_{\nu}\left(\varphi_{1}\right)+\sigma_{T}^{0}\left(\varphi_{2}\right): \partial_{m} \nu=f_{2 \nu} \text { sur } \Sigma_{T} \\
\varphi_{1}(0)=\varphi_{1}^{0}, \quad \varphi_{1}^{\prime}(0)=\varphi_{1}^{1} \quad \text { dans } \Omega \\
\varphi_{2}(0)=\varphi_{2}^{0}, \quad \varphi_{2}^{\prime}(0)=\varphi_{2}^{1} \quad \text { sur } \quad \Gamma
\end{array}\right.
$$

et on suppose que $\varphi^{0}=\left(\varphi_{1}^{0}, \varphi_{2}^{0}\right) \in \mathcal{V}$ et $\varphi^{1}=\left(\varphi_{1}^{1}, \varphi_{2}^{1}\right) \in \mathcal{H}$. L'énergie de la solution $\varphi=\left(\varphi_{1}, \varphi_{2}\right)$ est définie par:

$$
\begin{aligned}
& E(\varphi, t)=\frac{1}{2} \int_{\Omega}\left(\sigma\left(\varphi_{1}\right): \epsilon\left(\varphi_{1}\right)+\left|\varphi_{1}^{\prime}\right|^{2}\right) d x+ \\
& \frac{1}{2} \int_{\Gamma}\left(\sigma_{T}^{0}\left(\varphi_{2}\right): \epsilon_{T}^{0}\left(\varphi_{2}\right)+\left|\varphi_{2}^{\prime}\right|^{2}\right) d \Gamma
\end{aligned}
$$


On pose $f=\left(f_{1}, f_{2}\right)$ avec $f_{2}=f_{2 T}+f_{2 \nu} \nu$; si $f=0$ on dira que le problème (1.6) est homogène; l'énergie est alors une constante qui sera notée $E_{0}$.

Soit $\mathbf{x}_{0} \in \mathbb{R}^{3}$; on pose: $\mathbf{q}(\mathbf{x})=\mathbf{x}-\mathbf{x}_{0}$ et $R\left(\mathbf{x}_{0}\right)=\operatorname{Max}\left\{\left|\mathbf{x}-\mathbf{x}_{0}\right| ; x \in\right.$ $\bar{\Omega}\}$; on désigne par $\nabla_{T}$ le gradient tangentiel et par $\Delta_{T}$ le laplatien tangentiel; on démontre le théorème d'unicité suivant:

Theoreme 1.2. Soient $\varphi^{0}=\left(\varphi_{1}^{0}, \varphi_{2}^{0}\right) \in D(\mathcal{A})$ et $\varphi^{1}=\left(\varphi_{1}^{1}, \varphi_{2}^{1}\right) \in \mathcal{V}$. Il existe trois constantes positives $\delta$, $\gamma$ et $c_{1}$, qui ne dépendent que de $\Omega$ telles que si $T_{0}=2 R\left(\mathbf{x}_{0}\right)(\sqrt{\delta}+\sqrt{\gamma})$ alors l'énergie $E_{0}$ de la solution $\varphi=\left(\varphi_{1}, \varphi_{2}\right)$ du problème homogène (1.6) vérifie l'estimation:

$$
\begin{gathered}
\left(T-T_{0}\right) E_{0}+\frac{1}{R\left(\mathbf{x}_{0}\right) \sqrt{\gamma}}\left|\varphi_{1}^{0}\right|_{\Omega}^{2}+\frac{1}{2 R\left(\mathbf{x}_{0}\right) \sqrt{\delta}}\left|\varphi_{1}^{0}\right|_{\Gamma}^{2} \leq \\
c_{1}\left(\int_{\Sigma_{T}}\left(\left|\varphi_{2}\right|^{2}+\left|\varphi_{2}^{\prime}\right|^{2}+\sigma_{T}^{0}\left(\varphi_{2}\right): \epsilon_{T}^{0}\left(\varphi_{2}\right)+\left|\nabla_{T} \varphi_{1 \nu}\right|^{2}+\left|\sigma\left(\varphi_{1}\right) . \nu\right|^{2}\right) d \Sigma\right)
\end{gathered}
$$

Pour donner un sens au tenseur de courbure on suppose que la frontière $\Gamma$ de $\Omega$ est de classe $C^{3}$ (cf.[6]).

L'estimation (1.8) permet une mise en oeuvre de la méthode d'unicité hilbertienne, (H.U.M.), de J.L. Lions, (cf.[9]), qui conduit au résultat de contrôlabilité exacte suivant:

Theoreme 1.3. On suppose $\Gamma$ de classe $C^{3}$; il existe un temps $T_{0}=2 R\left(\mathbf{x}_{0}\right)(\sqrt{\delta}+\sqrt{\gamma})$ où $\delta$ et $\gamma$ sont deux constantes qui ne dépendent que de $\Omega$, un espace de Hilbert $\mathcal{F}$ et un isomorphisme $\Lambda: \mathcal{F} \hookrightarrow \mathcal{F}^{\prime}$ qui vérifient:

(i) Pour tout $\left(\rho^{1},-\rho^{0}\right) \in \mathcal{F}^{\prime}$ il existe un contrôle $\mathbf{g}=\mathbf{g}_{T}+g_{\nu} \nu$ tel que la solution $\mathbf{u} d u$ problème (1.5) avec $\mathbf{f}=0, T>T_{0}$ et les conditions initiales $\left(\mathbf{u}, \mathbf{u}_{\mid \Gamma}\right)(0)=\rho^{0}, \quad\left(\mathbf{u}, \mathbf{u}_{\mid \Gamma}\right)^{\prime}(0)=\rho^{1}$ vérifie: $\quad \mathbf{u}(T)=\mathbf{u}^{\prime}(T)=$ 0 .

(ii) On pose $\left(\varphi^{0}, \varphi^{1}\right)=\Lambda^{-1}\left(\rho^{1},-\rho^{0}\right)$ et on note $\varphi=\left(\varphi_{1}, \varphi_{2}\right), \quad\left(\varphi_{2}=\right.$ $\left.\varphi_{1 \mid \Sigma_{T}}\right)$ la solution du problème homogène (1.6) correspondant; alors le contrôle $\mathbf{g}$ est donné par:

$$
\left\{\begin{array}{l}
\mathbf{g}_{T}=\overline{\operatorname{div}_{T} \sigma_{T}^{0}}\left(\varphi_{1}\right)-\varphi_{1 T}+\partial_{t} \varphi_{1 T}^{\prime} \\
g_{\nu}=-\sigma_{T}^{0}\left(\varphi_{1}\right): \partial_{m} \nu-\varphi_{1 \nu}+\partial_{t} \varphi_{1 \nu}^{\prime}+\Delta_{T} \varphi_{1 \nu}
\end{array}\right.
$$


$\partial_{t}$ est la dérivation au sens de la dualité, (cf. remarque 5.4.)

Dans ce travail on adopte le plan suivant: Au paragraphe 2 on donne quelques notations et quelques résultats préliminaires de géométrie intrinsèque pour les surfaces de $\mathbb{R}^{3}$. Le paragraphe 3 est consacré à la démonstration du théorème 1.1. Au pragraphe 4 on établit une identié qui permet de montrer le théorème 1.2. Au paragraphe 5 on démontre le thérème 1.3 .

\section{Notations et résultats préliminaires}

On reprend les définitions et les notations données en introduction. La convention de l'indice répété est adoptée:

$\operatorname{tr}(\tau)=\tau_{11}+\tau_{22}+\ldots=\tau_{i i}, \mathbf{v} \cdot \mathbf{w}=v_{i} w_{i}, \sigma(\mathbf{v}): \epsilon(\mathbf{v})=\sigma_{i j}(\mathbf{v}) \epsilon_{i j}(\mathbf{v})$.

Comme $\Gamma$ est de classe $C^{2}$, pour tout point $m$ de $\Gamma$ on peut trouver un $C^{2}$-difféomorphisme $\chi$ d'un ouvert $\hat{\Gamma}$ de $\mathbb{R}^{2}$ (rapporté au système orthonormé $\left.\left(0, \xi^{1}, \xi^{2}\right)\right)$ sur un voisinage ouvert de $m$ dans $\Gamma$. Les vecteurs $\mathbf{a}_{\alpha}=\partial \chi / \partial \xi^{\alpha}\left(\chi^{-1}(m)\right), \alpha \in\{1,2\}$ engendrent le plan $\mathrm{T}_{m}(\Gamma)$ tangent en $m$ à $\Gamma$. On note par $T(\Gamma)$ le fibré tangent, $(c f .[1],[7],[12])$. Soit $\mathcal{G}$ le tenseur métrique associé à $\chi$ de composantes: $g_{\alpha \beta}=\mathbf{a}_{\alpha} \cdot \mathbf{a}_{\beta}, \forall(\alpha, \beta) \in$ $\{1,2\}^{2}$ et $\left(g^{\alpha \beta}\right)_{1 \leq \alpha, \beta \leq 2}$ le tenseur inverse. La base duale de $\left\{\mathbf{a}_{\alpha}\right\}_{\alpha=1,2}$ est définie par: $\mathbf{a}^{\alpha}(m) \cdot \mathbf{a}_{\beta}(m)=\delta_{\beta}^{\alpha}$, (symbole de Kreonecker).

A un champ tangent $\mathbf{v}_{T}=v^{\alpha} \mathbf{a}_{\alpha}$ on associe, par le produit scalaire de $\mathbb{R}^{3}$, le champ de formes linéaires: $\overline{\mathbf{v}_{T}}=v_{\alpha} \mathbf{a}^{\alpha}$ avec $v_{\alpha}=g_{\alpha \beta} v^{\beta}$.

Tout champ vectoriel $\mathbf{v}$ défini sur $\Gamma$ s'écrit: $\mathbf{v}=\mathbf{v}_{T}+v_{\nu} \nu$ où $\mathbf{v}_{T}(m)=$ $\pi(m) \mathbf{v}(m)$ est la projection orthogonale de $\mathbf{v}(m)$ sur $T_{m}(\Gamma)$ et $v_{\nu}(m)=\overline{\nu(m)} \mathbf{v}(m)$. Soit $\mathbf{v}_{T}(m)$ un champ régulier de vecteurs tangents défini sur $\Gamma$; on définit la dérivée $\partial_{m} \mathbf{v}_{T}$ de $\mathbf{v}_{T}$ sur $\Gamma$ par: $\partial_{m} v_{T}=$ $\left(\partial_{\alpha} v_{T}\right) a^{\alpha},\left(\partial_{\alpha}\right.$ est la dérivée par rapport à $\left.\xi^{\alpha}\right)$; c'est une application linéaire qui applique le plan tangent dans $\mathbb{R}^{3}$ et non dans lui- même. On définit alors les symboles de Cristoffel $\Gamma_{\alpha \beta}^{\lambda}$ par:

$$
\Gamma_{\alpha \beta}^{\lambda}=a^{\lambda} \pi a_{\alpha, \beta} \quad \text { avec } \quad a_{\alpha, \beta}=\partial_{\alpha \beta}^{2} \chi, \quad \alpha, \beta=1,2
$$

La dérivée covariante du champ $\mathbf{v}_{T}$ sur $\Gamma$ est $\pi \partial_{m} \mathbf{v}_{T} \pi$; elle applique le plan tangent dans lui- même; si $\mathbf{v}_{T}=v^{\alpha} a_{\alpha}$, la définition des symboles 
de Cristoffel montre que:

$$
\pi \partial_{m} \mathbf{v}_{T} \pi=\left(v_{, \alpha}^{\beta}+\Gamma_{\alpha \lambda}^{\beta} v^{\lambda}\right) a_{\beta} a^{\alpha} .
$$

On définit l'opérateur de courbure de $\Gamma$ par: $\partial_{m} \nu=\left(\partial_{\alpha} \nu\right) a^{\alpha}$; c'est un endomorphisme symétrique du plan tangent. Ses valeurs propres sont les courbures principales de $\Gamma$ (cf.[1],[7],[12]).

De $\bar{\nu} \mathbf{v}_{T}=0$ on obtient par dérivation $\bar{\nu}\left(\partial_{m} \mathbf{v}_{T}\right)+\overline{\mathbf{v}_{T}}\left(\partial_{m} \nu\right)=0$, ce qui permet d'écrire la dérivée de $\mathbf{v}$ sous la forme:

$$
\partial_{m} \mathbf{v}=\pi \partial_{m} \mathbf{v}_{T} \pi-\nu \overline{\mathbf{v}_{T}}\left(\partial_{m} \nu\right)+\nu\left(\partial_{m} v_{\nu}\right)+v_{\nu}\left(\partial_{m} \nu\right)
$$

A un champ scalaire régulier $w$ défini sur $\Gamma$ on associe le champ de formes linéaires du plan tangent défini par:

$$
\left(\partial_{m} w\right)(m)=w_{, \alpha}(m) \mathbf{a}^{\alpha}(m) .
$$

Le vecteur transposé de la forme $\left(\partial_{m} w\right)(m)$ est le gradient tangentiel noté $\left(\nabla_{T} w\right)(m)$.

\subsection{Déformations et contraintes}

Soit $\mathbf{v}: \bar{\Omega} \rightarrow \mathbb{R}^{3}$ un champ assez régulier; on désigne par $\nabla$ son gradient; on a sur $\Gamma(c f .[1],[7],[12])$ :

$$
\begin{gathered}
\mathrm{d} \mathbf{v}=\overline{\nabla \mathbf{v}}=\pi\left(\partial_{m} \mathbf{v}_{T}\right) \pi+v_{\nu}\left(\partial_{m} \nu\right)+\left(\partial_{\nu} \mathbf{v}_{T}\right) \bar{\nu}+ \\
\nu\left(\left(\partial_{m} v_{\nu}\right)-\overline{\mathbf{v}_{T}}\left(\partial_{m} \nu\right)+\left(\partial_{\nu} v_{\nu}\right) \bar{\nu}\right)
\end{gathered}
$$

Les relations (1.1)- (1.4) sont une conséquence directe de (2.1).

Remarque 2.1. Soit $\mathbf{v} \in \mathbb{H}^{1}(\Omega)$; des formules (1.1)-(1.4) on obtient:

$$
\begin{aligned}
\epsilon(\mathbf{v}): \epsilon(\mathbf{v}) & =\epsilon_{T}(\mathbf{v}): \epsilon_{T}(\mathbf{v})+2\left|\epsilon_{S}(\mathbf{v})\right|^{2}+\left|\epsilon_{\nu}(\mathbf{v})\right|^{2} ; \\
\sigma(\mathbf{v}): \epsilon(\mathbf{v}) & =2 \mu\left(\epsilon_{T}(\mathbf{v}): \epsilon_{T}(\mathbf{v})+\left|\epsilon_{\nu}(\mathbf{v})\right|^{2}\right)+4 \mu\left|\epsilon_{S}(\mathbf{v})\right|^{2} \\
& +\lambda\left(\operatorname{tr}\left(\epsilon_{T}(\mathbf{v})\right)+\epsilon_{\nu}(\mathbf{v})\right)^{2} .
\end{aligned}
$$

On aura à considérer les espaces:

$\mathcal{L}_{S}\left(T_{m}(\Gamma)\right)$ est l'espace des endomorphismes symétriques de $T_{m}(\Gamma)$.

$\mathcal{L}_{S}(T(\Gamma))$ est l'espace des opérateurs symétriques de $T(\Gamma)$; Un champ $\tau: \Gamma \rightarrow T(\Gamma)$ appartient à $\mathcal{L}_{S}(T(\Gamma))$ si $\tau(m)$ appartient à $\mathcal{L}_{S}\left(T_{m}(\Gamma)\right)$ pour tout $m \in \Gamma$.

Remarque 2.2. $\left(\partial_{m} \nu\right)(m)$ est un élément de $\mathcal{L}_{S}\left(T_{m}(\Gamma)\right)$; ses valeurs propres sont les courbures principales de $\Gamma$ en $m$. 


\subsection{Quelques espaces fonctionnels}

Soit $\mathbf{v}_{T}: \Gamma \rightarrow T(\Gamma), \mathbf{v}_{T}(m)=v^{\alpha}(m) \mathbf{a}_{\alpha}(m)$ un champ tangent; On munit $L^{2}(\Gamma, T(\Gamma))$ de la norme:

$$
\left\|\mathbf{v}_{T}\right\|_{L^{2}(\Gamma, T(\Gamma))}=\left(\int_{\Gamma}\left|\mathbf{v}_{T}\right|^{2} d \Gamma\right)^{\frac{1}{2}}
$$

qui est équivalente à la norme:

$$
\mathbf{v}_{T} \rightarrow\left(\left\|v^{1}\right\|_{L^{2}(\Gamma)}^{2}+\left\|v^{2}\right\|_{L^{2}(\Gamma)}^{2}\right)^{\frac{1}{2}} .
$$

On munit $H^{1}(\Gamma, T(\Gamma))$ de la norme:

$$
\left\|\mathbf{v}_{T}\right\|_{H^{1}(\Gamma, T(\Gamma))}=\left(\left\|v^{1}\right\|_{H^{1}(\Gamma)}^{2}+\left\|v^{2}\right\|_{H^{1}(\Gamma)}^{2}\right)^{\frac{1}{2}} .
$$

Un champ $\tau_{T}: \Gamma \rightarrow \mathcal{L}_{S}(T(\Gamma))$ appartient à $L^{2}\left(\Gamma, \mathcal{L}_{S}(T(\Gamma))\right)$ si $\left(\tau_{T}: \tau_{T}\right)^{\frac{1}{2}}: \Gamma \rightarrow \mathbb{R}^{+}$appartient à $L^{2}(\Gamma)$; on pose:

$$
\left\|\tau_{T}\right\|_{L^{2}\left(\Gamma, \mathcal{L}_{S}(T(\Gamma))\right)}=\left\|\left(\tau_{T}: \tau_{T}\right)^{\frac{1}{2}}\right\|_{L^{2}(\Gamma)}
$$

Remarque 2.3. Si $\mathbf{v}_{T} \in H^{1}(\Gamma, T(\Gamma))$, alors: $\epsilon_{T}\left(\mathbf{v}_{T}\right) \in L^{2}\left(\Gamma, \mathcal{L}_{S}(T(\Gamma))\right)$.

Proposition 2.1. L'expression $\|\cdot\|_{H^{1}(\Gamma, T(\Gamma))}^{1}$ définie par:

$$
\left\|\mathbf{v}_{T}\right\|_{H^{1}(\Gamma, T(\Gamma))}^{1}=\left(\int_{\Gamma}\left(\left|\mathbf{v}_{T}\right|^{2}+\epsilon_{T}^{0}\left(\mathbf{v}_{T}\right): \epsilon_{T}^{0}\left(\mathbf{v}_{T}\right)\right) d \Gamma\right)^{\frac{1}{2}}
$$

est une norme sur $H^{1}(\Gamma, T(\Gamma))$ équivalente à la norme définie en (2.2).

Preuve. Il suffit de prouver l'existence d'une constante $C>0$ telle que:

$$
\left\|\mathbf{v}_{T}\right\|_{H^{1}(\Gamma, T(\Gamma))} \leq C\left\|\mathbf{v}_{T}\right\|_{H^{1}(\Gamma, T(\Gamma))}^{1}, \quad \forall \mathbf{v}_{T} \in H^{1}(\Gamma, T(\Gamma))
$$

S'il n'en était pas ainsi, il existerait une suite $\left\{\mathbf{v}_{T}^{k}\right\} \subset H^{1}(\Gamma, T(\Gamma))$ vérifiant:

$$
\begin{array}{cccc}
\left\|\mathbf{v}_{T}^{k}\right\|_{H^{1}(\Gamma, T(\Gamma))}=1, & \forall k \in \mathbb{I N}, & \mathbf{v}_{T}^{k} \longrightarrow 0 \quad \text { dans } & L^{2}(\Gamma, T(\Gamma)) \\
\epsilon_{T}\left(\mathbf{v}_{T}^{k}\right) \longrightarrow 0 \quad \text { dans } & L^{2}\left(\Gamma, L_{S}(T(\Gamma))\right) &
\end{array}
$$


On pose: $\mathbf{v}_{T}^{k}=v_{T}^{k 1} \mathbf{a}_{1}+v_{T}^{k 2} \mathbf{a}_{2}$; les expressions de $\pi \partial_{m} \mathbf{v}_{T} \pi$ et du conjugué d'un vecteur données au début de ce paragraphe montrent que:

$\overline{\pi \partial_{m} \mathbf{v}_{T} \pi}=\left(v_{, \lambda}^{k \mu}+\Gamma_{\lambda r}^{\mu} v^{k r}\right) g^{\lambda \alpha} g_{\mu \beta} \mathbf{a}_{\alpha} \mathbf{a}^{\beta}$, d'où: $\left(v_{, \beta}^{k \alpha}+g^{\lambda \alpha} g_{\beta \mu} v_{, \lambda}^{k \mu}\right) \rightarrow 0$ dans $L^{2}(\Gamma)$. En prenant $\alpha=\beta$, on obtient: $v_{, 1}^{k 1}+v_{, 2}^{k 2} \rightarrow 0$ dans $L^{2}(\Gamma)$ On a: $g_{\alpha \eta}\left(v_{, \beta}^{k \alpha}+g^{\lambda \alpha} g_{\beta \mu} v_{, \lambda}^{k \mu}\right)=g_{\alpha \eta} v_{, \beta}^{k \alpha}+g_{\beta \mu} v_{, \eta}^{k \mu} \rightarrow 0$ dans $L^{2}(\Gamma)$.

En prenant $(\beta, \eta)=(1,1),(\beta, \eta)=(2,2)$ et $(\beta, \eta)=(1,2)$ on obtient les limites suivantes dans $L^{2}(\Gamma):\left(g_{11} v_{, 1}^{k 1}+g_{21} v_{, 1}^{k 2}\right) \rightarrow 0,\left(g_{22} v_{, 2}^{k 2}+g_{21} v_{, 2}^{k 1}\right) \rightarrow$ $0,\left(g_{11} v_{, 2}^{k 1}+g_{22} v_{, 1}^{k 2} \rightarrow 0\right.$. Posons: $\mathbf{w}_{T}^{k}=\mathcal{G} \mathbf{v}_{T}^{k}=w^{k 1} \mathbf{a}_{1}+w^{k 2} \mathbf{a}_{2}$. De $\mathbf{v}_{T}^{k} \rightarrow$ 0 dans $L^{2}(\Gamma, T(\Gamma))$ et des limites précédentes on déduit que les suites $\left(w^{k 1}\right),\left(w^{k 2}\right),\left(w_{, 1}^{k 1}\right),\left(w_{, 2}^{k 2}\right),\left(w_{, 1}^{k 2}+w_{, 2}^{k 1}\right)$ tendent vers 0 dans $L^{2}(\Gamma)$.

L'inégalitée de Korn dans l'ouvert $\hat{\Gamma}$ montre alors que $\left(w^{k 1}, w^{k 2}\right) \rightarrow 0$ dans $H^{1}(\Gamma) \times H^{1}(\Gamma)$. D'où $\left(v^{k 1}, v^{k 2}\right) \rightarrow 0$ dans $\quad H^{1}(\Gamma) \times H^{1}(\Gamma)$ ce qui contredit la définition de la suite $\left\{\mathbf{v}_{T}^{k}\right\}$.

Proposition 2.2. L'application: $\mathbf{v}_{T} \hookrightarrow \mathbf{v}_{T}-\overline{\operatorname{div} v_{T} \sigma_{T}^{0}\left(\mathbf{v}_{T}\right)}$ est un isomorphisme de $H^{s}(\Gamma, T(\Gamma))$ sur $H^{s-2}(\Gamma, T(\Gamma))$ pour tout réel $s \geq 1$.

Preuve. La forme bilinéaire:

$$
\mathcal{B}:\left(\mathbf{v}_{T}, \mathbf{w}_{T}\right) \hookrightarrow \int_{\Gamma}\left(\mathbf{v}_{T} \cdot \mathbf{w}_{T}+\sigma_{T}^{0}\left(\mathbf{v}_{T}\right): \epsilon_{T}^{0}\left(\mathbf{w}_{T}\right)\right) d \Gamma
$$

est continue et coercive sur $H^{1}(\Gamma, T(\Gamma))=\left(H_{0}^{1}(\Gamma, T(\Gamma))\right)$ d'après la proposition 2.1; alors pour tout $f \in H^{-1}(\Gamma, T(\Gamma))$ il existe un unique $\mathbf{v}_{T} \in H^{1}(\Gamma, T(\Gamma))$ tel que: $\mathcal{B}\left(\mathbf{v}_{T}, \mathbf{w}_{T}\right)=\left(f, \mathbf{w}_{T}\right), \quad \forall \mathbf{w}_{T} \in H^{1}(\Gamma, T(\Gamma))$. avec:

$$
C_{2}\|f\|_{H^{-1}(\Gamma, T(\Gamma))} \leq\left\|\mathbf{v}_{T}\right\|_{H^{1}(\Gamma, T(\Gamma))} \leq C_{1}\|f\|_{H^{-1}(\Gamma, T(\Gamma))}
$$

ce qui prouve que l'application $\mathbf{v}_{T} \hookrightarrow \mathbf{v}_{T}-\overline{\operatorname{div}_{T} \sigma_{T}^{0}\left(\mathbf{v}_{T}\right)}$ est un isomorphisme de $H^{1}(\Gamma, T(\Gamma))$ sur $H^{-1}(\Gamma, T(\Gamma))$.

L'opérateur $\mathbf{v}_{T} \hookrightarrow \overline{\operatorname{div}_{T} \sigma_{T}^{0}\left(\mathbf{v}_{T}\right)}$ étant elliptique, cette application est aussi un isomorphisme de $H^{2}(\Gamma, T(\Gamma))$ sur $L^{2}(\Gamma, T(\Gamma))$. comme $H^{1}(\Gamma, T(\Gamma))=H_{0}^{1}(\Gamma, T(\Gamma))$ on déduit par interpolation que cette application est un isomorphisme de $H^{2-s}(\Gamma, T(\Gamma))$ sur $H^{-s}(\Gamma, T(\Gamma))$ pour tout réel $s$ compris entre 0 et 1, $(c f .[10])$. On termine par interpolation grâce à l'ellipticité de l'opérateur $\mathbf{v}_{T} \hookrightarrow \overline{\operatorname{div}_{T} \sigma_{T}^{0}\left(\mathbf{v}_{T}\right)}$. 


\subsection{Densité et régularité}

Lemme 2.1. Soit $H($ divo $)$ l'espace défini par:

$$
H(\operatorname{div} \sigma)=\left\{v \in \mathbb{L}^{2}(\Omega): \operatorname{div} \sigma(v) \in \mathbb{L}^{2}(\Omega)\right\}
$$

muni de la norme:

$$
\|v\|_{H(d i v \sigma)}=\left(\|v\|_{\mathbb{L}^{2}(\Omega)}^{2}+\|\operatorname{div} \sigma(v)\|_{\mathbb{L}^{2}(\Omega)}^{2}\right)^{\frac{1}{2}}
$$

On peut définir une application continue

$$
H(\operatorname{div} \sigma) \longrightarrow \mathbb{H}^{-\frac{1}{2}}(\Gamma) \times \mathbb{H}^{-\frac{3}{2}}(\Gamma), \quad v \longrightarrow\left(v_{\mid \Gamma},(\sigma(v) . \nu)_{\mid \Gamma}\right)
$$

Preuve. Soient $w \in \mathbb{H}^{\frac{3}{2}}(\Gamma)$ et $\tilde{w} \in \mathbb{H}^{2}(\Omega)$ (un relèvement continu) vérifiant $\tilde{w}_{\mid \Gamma}=w$ et $(\sigma(\tilde{w}) . \nu)_{\mid \Gamma}=0$. Pour $v \in H(\operatorname{div} \sigma)$ on pose:

$$
\left(\tilde{\gamma_{1}} v, w\right)=\int_{\Omega}(\tilde{w} \operatorname{div} \sigma(v)-v \operatorname{div} \sigma(\tilde{w})) d x .
$$

On montre que le terme de droite ne dépend pas du relèvement $\tilde{w}$; il suffit pour cela de prouver que ce terme est nul si $\tilde{w}$ est un élément de $\mathbb{H}_{0}^{2}(\Omega)$.

Ce terme est nul si $\tilde{w}$ est un élément de $D\left(\Omega, \mathbb{R}^{3}\right)$ et par densité il sera nul pour tout élément de $\mathbb{H}_{0}^{2}(\Omega)$. On a:

$$
\left|\left(\tilde{\gamma_{1}} v, w\right)\right| \leq C\|w\|_{\mathbb{H}^{\frac{3}{2}(\Gamma)}}\|v\|_{H(\operatorname{div} \sigma)} .
$$

$\tilde{\gamma_{1}} v$ est donc un élément de $\mathbb{H}^{-\frac{3}{2}}(\Gamma)$ et on a

$$
\left\|\tilde{\gamma_{1}} v\right\|_{\mathbb{H}^{-\frac{3}{2}(\Gamma)}} \leq C\|v\|_{H(\operatorname{div} \sigma)} .
$$

$\tilde{\gamma_{1}}$ est donc continue de $H(\operatorname{div} \sigma)$ dans $\mathbb{H}^{-\frac{3}{2}}(\Gamma)$ et si $v$ est régulière on a $\tilde{\gamma_{1}} v=(\sigma(v) \cdot \nu)_{\mid \Gamma} \cdot$

Soient $w \in \mathbb{H}^{\frac{1}{2}}(\Gamma)$ et $\tilde{w} \in \mathbb{H}^{2}(\Omega)$ (un relèvement continu) vérifiant $\tilde{w}_{\mid \Gamma}=$ 0 et $(\sigma(\tilde{w}) \cdot \nu)_{\mid \Gamma}=w$. Pour $v \in H(\operatorname{div} \sigma)$ on pose:

$$
\left(\gamma_{0} v, w\right)=\int_{\Omega}(v \operatorname{div} \sigma(\tilde{w})-\tilde{w} \operatorname{div} \sigma(v)) d x .
$$


On vérifie, comme précédemment, que $\gamma_{0}$ est bien définie et on a:

$$
\left|\left(\gamma_{0} v, w\right)\right| \leq C\|w\|_{\mathbb{H}^{\frac{1}{2}(\Gamma)}}\|v\|_{H(\operatorname{div} \sigma)} .
$$

$\gamma_{0} v$ est donc un élément de $\mathbb{H}^{-\frac{1}{2}}(\Gamma)$ et on a:

$$
\left\|\gamma_{0} v\right\|_{\mathbb{H}^{-\frac{1}{2}(\Gamma)}} \leq C\|v\|_{H(\operatorname{div} \sigma)}
$$

$\gamma_{0}$ est donc continue de $H(\operatorname{div} \sigma)$ dans $\mathbb{H}^{-\frac{1}{2}}(\Gamma)$ et si $v$ est régulière on a $\gamma_{0} v=v_{\mid \Gamma}$.

Remarque 2.4. Pour tout élément $w \in \mathbb{H}^{2}(\Omega)$ et tout élément $v \in$ $H(\operatorname{div} \sigma)$ on $a$ :

$$
\begin{gathered}
\langle v, \sigma(w) \cdot \nu\rangle_{\mathbb{H}^{-\frac{1}{2}}(\Gamma), \mathbb{H}^{\frac{1}{2}}(\Gamma)}-\langle\sigma(v) \cdot \nu, w\rangle_{\mathbb{H}^{-\frac{3}{2}}(\Gamma), \mathbb{H}^{\frac{3}{2}}(\Gamma)}= \\
\int_{\Omega}(v \operatorname{div} \sigma(w)-w \operatorname{div} \sigma(v)) d x
\end{gathered}
$$

Lemme 2.2. Soit $\mathbb{H}^{1}(\operatorname{div} \sigma)=\left\{v \in \mathbb{H}^{1}(\Omega)\right.$ : $\left.\operatorname{div} \sigma(v) \in \mathbb{L}^{2}(\Omega)\right\}$ muni de la norme:

$$
\|v\|_{\mathbb{H}^{1}(\operatorname{div} \sigma)}=\left(\|v\|_{\mathbb{H}^{1}(\Omega)}^{2}+\|\operatorname{div\sigma }(v)\|_{\mathbb{L}^{2}(\Omega)}^{2}\right)^{\frac{1}{2}}
$$

$\mathcal{D}\left(\bar{\Omega}, \mathbb{R}^{3}\right)$ est dense dans $\mathbb{H}^{1}($ div $\sigma)$

Preuve. Soit $v \in \mathbb{H}^{1}(\operatorname{div} \sigma)$, et $f=v-\operatorname{div} \sigma(v) \in \mathbb{L}^{2}(\Omega)$; on considère $\left\{f_{k}\right\} \subset \mathcal{D}\left(\bar{\Omega}, \mathbb{R}^{3}\right)$ telle que: $f_{k} \longrightarrow f$ dans $\mathbb{L}^{2}(\Omega)$ et $\left\{g_{k}\right\} \subset \mathcal{D}\left(\Gamma, \mathbb{R}^{3}\right)$ telle que: $g_{k} \longrightarrow v_{\mid \Gamma}$ dans $H^{\frac{1}{2}}\left(\Gamma, \mathbb{R}^{3}\right)$. Soit $v^{k}$ la solution du problème:

$$
\left\{\begin{array}{l}
v^{k}-\operatorname{div} \sigma\left(v^{k}\right)=f_{k} \text { dans } \Omega, \\
v_{\mid \Gamma}^{k}=g_{k} \text { sur } \Gamma .
\end{array}\right.
$$

On a: $\left\{v^{k}\right\} \subset \mathcal{D}\left(\bar{\Omega}, \mathbb{R}^{3}\right), v^{k} \longrightarrow v$ dans $\mathbb{H}^{1}(\Omega)$ et $\operatorname{div} \sigma\left(v^{k}\right) \longrightarrow \operatorname{div} \sigma(v)$ dans $\mathbb{L}^{2}(\Omega)$ donc $v^{k} \longrightarrow v$ dans $\mathbb{H}^{1}($ div $\sigma)$.

Corollaire 2.1. L'application:

$$
\begin{aligned}
& \mathcal{L}: \mathcal{D}\left(\bar{\Omega}, \mathbb{R}^{3}\right) \hookrightarrow \mathbb{H}^{-\frac{1}{2}}(\Gamma) \\
& v \hookrightarrow \sigma(v) \nu=\sigma_{S}(v)+\sigma_{\nu}(v) \nu
\end{aligned}
$$

se prolonge de façon unique en une application continue:

$$
\mathbb{H}^{1}(\operatorname{div} \sigma) \hookrightarrow \mathbb{H}^{-\frac{1}{2}}(\Gamma)
$$


Preuve. $\mathcal{L}$ est définie sur $\mathcal{D}\left(\bar{\Omega}, \mathbb{R}^{3}\right)$ par:

$$
(\mathcal{L} u, v)=\int_{\Omega}(\operatorname{div} \sigma(u) \cdot \tilde{v}+\sigma(u): \epsilon(\tilde{v})) d x
$$

où $v \hookrightarrow \tilde{v}$ est un relèvement continu de $\mathbb{H}^{\frac{1}{2}}(\Gamma)$ dans $\mathbb{H}^{1}(\Omega)$; il existe alors une constante positive $C$ telle que:

$$
|(\mathcal{L} u, v)| \leq C\left(\|u\|_{\mathbb{H}^{1}(\Omega)}+\|\operatorname{div} \sigma(u)\|_{\left.\mathbb{L}^{2}(\Omega)\right)}\right)\|v\|_{\mathbb{H}^{\frac{1}{2}}(\Gamma)}
$$

On applique ensuite le lemme 2.2.

Proposition 2.3. L'expression $\left|\|\cdot \mid\|_{\mathbb{V}}\right.$ définie par:

$$
\|\mathbf{v} \mid\|_{\mathbb{V}}=\left(\int_{\Omega}\left(\sigma(\mathbf{v}): \epsilon(\mathbf{v})+|\mathbf{v}|^{2}\right) d x+\int_{\Gamma} \sigma_{T}^{0}(\mathbf{v}): \epsilon_{T}^{0}(\mathbf{v}) d \Gamma\right)^{\frac{1}{2}}
$$

est une norme sur $\mathbb{V}$ équivalente à $\|\cdot\|_{\mathbb{V}}$ définie par:

$$
\|\mathbf{v}\|_{\mathbb{V}}=\left(\|\mathbf{v}\|_{\mathbb{H}^{1}(\Omega)}^{2}+\left\|\mathbf{v}_{T}\right\|_{H^{1}(\Gamma, T(\Gamma))}^{2}\right)^{\frac{1}{2}}
$$

Preuve. On montre qu'il existe $C>0$ tel que:

$$
\|\mathbf{v}\|_{\mathbb{V}} \leq C \mid\|\mathbf{v}\|_{\mathbb{V}}, \quad \forall \mathbf{v} \in \mathbb{V}
$$

Dans le cas contraire on aurait une suite $\left\{\mathbf{v}^{k}\right\} \subset \mathbb{V}$ qui vérifie:

$$
\left\|\mathbf{v}^{k}\right\|_{\mathbb{H}^{1}(\Omega)}+\left\|\mathbf{v}_{T}^{k}\right\|_{H^{1}(\Gamma, T(\Gamma))}=1, \quad \text { et } \quad\left|\left\|\mathbf{v}^{k} \mid\right\|_{\mathbb{V}} \rightarrow 0\right.
$$

De l'inégalité de Korn il vient: $\mathbf{v}^{k} \rightarrow 0$ dans $\mathbb{H}^{1}(\Omega)$. Alors $\mathbf{v}_{T}^{k} \rightarrow 0$ dans $L^{2}(\Gamma, T(\Gamma))$ et $v_{\nu}^{k} \rightarrow 0$ dans $L^{2}(\Gamma)$ ce qui joint à: $\int_{\Gamma} \sigma_{T}^{0}\left(\mathbf{v}^{k}\right)$ : $\epsilon_{T}^{0}\left(\mathbf{v}^{k}\right) d \Gamma \rightarrow 0$ donne: $\epsilon_{T}^{0}\left(\mathbf{v}_{T}^{k}\right) \rightarrow 0$ dans $L^{2}\left(\Gamma, L_{S}(T(\Gamma))\right)$. La proposition 2.1 montre que: $\mathbf{v}_{T}^{k} \rightarrow 0$ dans $H^{1}(\Gamma, T(\Gamma))$ ce qui avec $\mathbf{v}^{k} \rightarrow 0$ dans $\mathbb{H}^{1}(\Omega)$ donne une contradiction.

Proposition 2.4. L'expression $\|$. \| définie par:

$$
\|\mathbf{v}\|=\left(\int_{\Omega} \sigma(\mathbf{v}): \epsilon(\mathbf{v}) d x+\int_{\Gamma}\left(\sigma_{T}^{0}(\mathbf{v}): \epsilon_{T}^{0}(\mathbf{v})+|\mathbf{v}|^{2}\right) d \Gamma\right)^{\frac{1}{2}}
$$

est une norme sur $\mathbb{V}$ équivalente à $\|.\|_{\mathbb{V}}$ définie par:

$$
\|\mathbf{v}\|_{\mathbb{V}}=\left(\|\mathbf{v}\|_{\mathbb{H}^{1}(\Omega)}^{2}+\left\|\mathbf{v}_{T}\right\|_{H^{1}(\Gamma, T(\Gamma))}^{2}\right)^{\frac{1}{2}}
$$


Preuve. On sait de [9] qu'il existe $\delta>0$ tel que:

$$
\int_{\Omega}|\nabla \mathbf{v}|^{2} d x \leq \delta\left(\int_{\Omega} \sigma(\mathbf{v}): \epsilon(\mathbf{v}) d x+\int_{\Gamma}|\mathbf{v}|^{2} d \Gamma\right), \quad \forall \mathbf{v} \in \mathbb{H}^{1}(\Omega) .
$$

Ce qui montre que $\|$.$\| est bien une norme sur \mathbb{V}$; pour montrer qu'elle est équivalente à $\|.\| \mathbb{V}$ on suppose le contraire; nous aurons une suite $\left\{\mathbf{v}^{k}\right\} \subset \mathbb{V}$ qui vérifie:

$$
\left\{\begin{array}{l}
\left\|\mathbf{v}^{k}\right\|_{\mathbb{H}^{1}(\Omega)}+\left\|\mathbf{v}_{T}^{k}\right\|_{H^{1}(\Gamma, T(\Gamma))}=1 \\
\left\|\mathbf{v}^{k}\right\| \longrightarrow 0
\end{array}\right.
$$

Alors $\mathbf{v}_{\mid \Gamma}^{k} \rightarrow 0$ dans $\mathbb{L}^{2}(\Gamma)$ et $\epsilon_{T}^{0}\left(\mathbf{v}_{T}^{k}\right) \rightarrow 0$ dans $L^{2}\left(\Gamma, L_{S}(T(\Gamma))\right)$. la proposition 2.1 montre alors que: $\mathbf{v}_{T}^{k} \rightarrow 0$ dans $H^{1}(\Gamma, T(\Gamma))$. Comme l'injection de $\mathbb{H}^{1}(\Omega)$ dans $\mathbb{L}^{2}(\Omega)$ est compacte on obtient: $\mathbf{v}^{k} \rightarrow 0$ dans $\mathbb{L}^{2}(\Omega)$ (il s'agit en fait d'une sous suite). L'inégalité de Korn montre alors que: $\mathbf{v}^{k} \rightarrow 0$ dans $\mathbb{H}^{1}(\Omega)$ ce qui joint à $\mathbf{v}_{T}^{k} \rightarrow 0$ dans $H^{1}(\Gamma,(T(\Gamma)))$ donne une contradiction.

Proposition 2.5. On donne $\mathbf{u}_{T} \in H^{\frac{3}{2}}(\Gamma, T(\Gamma))$ et $w_{\nu} \in H^{\frac{1}{2}}(\Gamma)$. Il existe $\mathbf{v} \in \mathbb{H}^{2}(\Omega)$ vérifiant: $\mathbf{v}_{T}=\mathbf{u}_{T}, \sigma_{\nu}(\mathbf{v})=w_{\nu}$ sur $\Gamma$ et il existe une constante positive $C$ ne dépendant que de $\Omega$ telle que:

$$
\|\mathbf{v}\|_{\mathbb{H}^{2}(\Omega)} \leq C\left(\left\|\mathbf{u}_{T}\right\|_{H^{\frac{3}{2}(\Gamma, T(\Gamma))}}+\left\|w_{\nu}\right\|_{H^{\frac{1}{2}(\Gamma)}}\right) .
$$

Preuve. Soit $\mathbf{w}_{1}$ la solution du problème:

$$
\left\{\begin{array}{l}
\mathbf{w}_{1}-\operatorname{div} \sigma\left(\mathbf{w}_{1}\right)=0 \text { dans } \Omega \\
\mathbf{w}_{1 T}=\mathbf{u}_{T}, \quad w_{1 \nu}=0 \text { sur } \Gamma .
\end{array}\right.
$$

Alors $\mathbf{w}_{1} \in \mathbb{H}^{2}(\Omega)$ et $\quad\left\|\mathbf{w}_{1}\right\|_{H^{2}\left(\Omega, \mathbb{R}^{3}\right)} \leq C\left\|\mathbf{u}_{T}\right\|_{H^{\frac{3}{2}}(\Gamma, T(\Gamma))}$.

On a: $\sigma(\mathbf{w}) . \nu=\sigma_{S}(\mathbf{w})+\sigma_{\nu}(\mathbf{w}) \nu$; soit $\mathbf{w}_{2}$ la solution du problème:

$$
\left\{\begin{array}{l}
\mathbf{w}_{2}-\operatorname{div} \sigma\left(\mathbf{w}_{2}\right)=0 \text { dans } \Omega \\
\sigma\left(\mathbf{w}_{2}\right) \cdot \nu=\left(w_{\nu}-\sigma_{\nu}\left(\mathbf{w}_{1}\right)\right) \nu \text { sur } \Gamma
\end{array}\right.
$$

Alors $\mathbf{w}_{2} \in \mathbb{H}^{2}(\Omega)$ et

$$
\left\|\mathbf{w}_{2}\right\|_{\mathbb{H}^{2}(\Omega)} \leq C\left\|w_{\nu}-\sigma_{\nu}\left(\mathbf{w}_{1}\right)\right\|_{H^{\frac{1}{2}}(\Gamma)} .
$$

Pour $\eta$ positif assez petit on pose: 


$$
\Omega^{\eta}=\left\{M: O M=O m+x_{\nu} \nu(m), m \in \Gamma,-\eta<x_{\nu}<0\right\}
$$

Soit $\chi \in \mathcal{C}^{\infty}([-\eta, 0])$ telle que: $\chi=1$ au voisinage de zéro et $\chi=0$ au voisinage de $-\eta$. Si $\mathbf{w}_{2 T}=w_{2}^{\alpha} \mathbf{a}_{\alpha}$ on considère $\widetilde{w_{2}^{\alpha}}$ un relèvement continu de $w_{2}^{\alpha}$ dans $\mathbb{H}^{2}(\Omega)$ puis on pose: $\mathbf{w}_{3 T}\left(m, x_{\nu}\right)=\chi\left(x_{\nu}\right) \widetilde{w_{2}^{\alpha}}\left(m, x_{\nu}\right) \mathbf{a}_{\alpha}$ et $\mathbf{w}_{3}=\mathbf{w}_{3 T}$ dans $\Omega^{\eta}$ et zéro ailleurs. Alors $\mathbf{w}_{3} \in \mathbb{H}^{2}(\Omega)$ et

$$
\left\|\mathbf{w}_{3}\right\|_{\mathbb{H}^{2}(\Omega)} \leq C\left\|\mathbf{w}_{2}\right\|_{\mathbb{H}^{2}(\Omega)} \leq C\left\|w_{\nu}-\sigma_{\nu}\left(\mathbf{w}_{1}\right)\right\|_{H^{\frac{1}{2}}(\Gamma)}
$$

Soit $w_{4 \nu}$ un élément de $H^{2}(\Omega)$ tel que:

$$
\begin{array}{r}
w_{4 \nu \mid \Gamma}=0 ;\left(\partial_{\nu} w_{4 \nu}\right)_{\mid \Gamma}=\lambda(2 \mu+\lambda)^{-1} \operatorname{tr}\left(\pi \partial_{m} \mathbf{w}_{3 T} \pi\right)_{\mid \Gamma} \text { et } \\
\left\|w_{4 \nu}\right\|_{H^{2}(\Omega)} \leq C\left\|\operatorname{tr}\left(\pi \partial_{m} \mathbf{w}_{3 T}\right)\right\|_{H^{\frac{1}{2}}(\Gamma)}
\end{array}
$$

On pose: $\mathbf{w}_{4}\left(m, x_{\nu}\right)=\chi\left(x_{\nu}\right) w_{4 \nu}\left(m, x_{\nu}\right) \nu(m)$ pour $-\eta<x_{\nu}<0$ et $\mathbf{w}_{4}=0$ ailleurs. Alors: $\left\|\mathbf{w}_{4}\right\|_{\mathbb{H}^{2}(\Omega)} \leq C\left\|w_{\nu}-\sigma_{\nu}\left(\mathbf{w}_{1}\right)\right\|_{H^{\frac{1}{2}}(\Gamma)}$.

On termine la démonstration en prenant: $\mathbf{v}=\mathbf{w}_{1}+\mathbf{w}_{2}-\mathbf{w}_{3}+\mathbf{w}_{4}$.

Proposition 2.6. Soit $\mathbf{u} \in \mathbb{V}$ avec $\operatorname{div} \sigma(\mathbf{u}) \in \mathbb{L}^{2}(\Omega), \mathbf{u}_{T} \in H^{\frac{3}{2}}(\Gamma, T(\Gamma))$ et $\sigma_{\nu}(\mathbf{u}) \in H^{\frac{1}{2}}(\Gamma)$. Alors: $\mathbf{u} \in \mathbb{H}^{2}(\Omega)$ et il existe une constante positive $C$ telle que:

$$
\|\mathbf{u}\|_{\mathbb{H}^{2}(\Omega)} \leq C\left(\|\mathbf{u}-\operatorname{div} \sigma(\mathbf{u})\|_{\mathbb{L}^{2}(\Omega)}+\left\|\mathbf{u}_{T}\right\|_{H^{\frac{3}{2}}(\Gamma, T(\Gamma))}+\left\|\sigma_{\nu}(\mathbf{u})\right\|_{H^{\frac{1}{2}}(\Gamma)}\right)
$$

Preuve. D'après la proposition 2.5 il existe $\mathbf{v} \in \mathbb{H}^{2}(\Omega)$ tel que $\mathbf{v}_{T}=$ $\mathbf{u}_{T}, \sigma_{\nu}(\mathbf{v})=\sigma_{\nu}(\mathbf{u})$ et

$$
\|\mathbf{v}\|_{\mathbb{H}^{2}(\Omega)} \leq C\left(\left\|\mathbf{u}_{T}\right\|_{H^{\frac{3}{2}}(\Gamma, T(\Gamma))}+\left\|\sigma_{\nu}(\mathbf{u})\right\|_{H^{\frac{1}{2}}(\Gamma)}\right) .
$$

On pose: $\mathbf{u}=(\mathbf{u}-\mathbf{v})+\mathbf{v}$ et $\mathbf{w}=\mathbf{u}-\mathbf{v} ;$ on a: $\mathbf{w}_{T}=0, \sigma_{\nu}(\mathbf{w})_{\mid \Gamma}=$ 0 et $\operatorname{div} \sigma(\mathbf{w}) \in \mathbb{L}^{2}(\Omega)$; alors: $\mathbf{w} \in \mathbb{H}^{2}(\Omega)$, et $\|\mathbf{w}\|_{\mathbb{H}^{2}(\Omega)} \leq C \| \mathbf{w}-$ $\operatorname{div} \sigma(\mathbf{w}) \|_{\mathbb{L}^{2}(\Omega)},(c f .[11])$, ce qui donne:

$$
\begin{gathered}
\|\mathbf{u}\|_{\mathbb{H}^{2}(\Omega)} \leq\|\mathbf{v}\|_{\mathbb{H}^{2}(\Omega)}+\|\mathbf{w}\|_{\mathbb{H}^{2}(\Omega)} \leq \\
C\left(\|\mathbf{u}-\operatorname{div} \sigma(\mathbf{u})\|_{\mathbb{L}^{2}(\Omega)}+\left\|\mathbf{u}_{T}\right\|_{H^{\frac{3}{2}}(\Gamma, T(\Gamma))}+\left\|\sigma_{\nu}(\mathbf{u})\right\|_{H^{\frac{1}{2}(\Gamma)}}\right)
\end{gathered}
$$


Proposition 2.7. Soit

$$
\begin{aligned}
\mathcal{Z}=\{\mathbf{u} \in \mathbb{V} & : \operatorname{div} \sigma(\mathbf{u}) \in \mathbb{L}^{2}(\Omega), \sigma_{S}(\mathbf{u})-\overline{\operatorname{div}_{T} \sigma_{T}^{0}}(\mathbf{u}) \\
& \in H^{\frac{1}{2}}\left(\Gamma, T(\Gamma), \sigma_{\nu}(\mathbf{u})+\sigma_{T}^{0}(\mathbf{u}): \partial_{m} \nu \in H^{\frac{1}{2}}(\Gamma)\right\} ;
\end{aligned}
$$

on a $\mathcal{Z} \subset \mathbb{H}^{2}(\Omega), \quad \mathbf{u}_{T} \in H^{\frac{5}{2}}(\Gamma, T(\Gamma)) \quad \forall \mathbf{u} \in \mathcal{Z} \quad$ et il existe une constante positive $C$ ne dépendant que de $\Omega$ telle que:

$$
\begin{gathered}
\|\mathbf{u}\|_{\mathbb{H}^{2}(\Omega)}+\left\|\mathbf{u}_{T}\right\|_{H^{\frac{5}{2}}(\Gamma, T(\Gamma))} \leq C\left\|\sigma_{S}(\mathbf{u})-\overline{\operatorname{div}_{T} \sigma_{T}^{0}}(\mathbf{u})\right\|_{H^{\frac{1}{2}}(\Gamma, T(\Gamma))} \\
+C\left(\|\mathbf{u}-\operatorname{div} \sigma(\mathbf{u})\|_{\mathbb{L}^{2}(\Omega)}+\left\|\sigma_{\nu}(\mathbf{u})+\sigma_{T}^{0}(\mathbf{u}): \partial_{m} \nu\right\|_{H^{\frac{1}{2}}(\Gamma)}\right), \quad \forall \mathbf{u} \in \mathcal{Z}
\end{gathered}
$$

Preuve. Soit $\mathbf{u} \in \mathcal{Z}$; d'après le corollaire 2.1 on a

$\sigma_{S}(\mathbf{u}) \in H^{-\frac{1}{2}}(\Gamma, T(\Gamma)) ; \operatorname{de} \sigma_{T}^{0}(\mathbf{u})=\sigma_{T}^{0}\left(\mathbf{u}_{T}\right)+2 \mu u_{\nu} \partial_{m} \nu+\lambda^{*} u_{\nu} \operatorname{tr}\left(\partial_{m} \nu\right) I_{2}$ il vient alors

$\overline{\operatorname{div}_{T} \sigma_{T}^{0}\left(\mathbf{u}_{T}\right)} \in H^{-\frac{1}{2}}(\Gamma, T(\Gamma))$ et donc $\mathbf{u}_{T} \in H^{\frac{3}{2}}(\Gamma, T(\Gamma))$ d'après la proposition 2.2; on obtient $\sigma_{\nu}(\mathbf{u}) \in H^{\frac{1}{2}}(\Gamma)$ et donc $\mathbf{u} \in \mathbb{H}^{2}(\Omega)$ d'après la proposition 2.6 et il existe une constante positive $C$ ne dépendant que de $\Omega$ telle que:

$$
\|\mathbf{u}\|_{\mathbb{H}^{2}(\Omega)} \leq C\left(\|\mathbf{u}-\operatorname{div} \sigma(\mathbf{u})\|_{\mathbb{L}^{2}(\Omega)}+\left\|\mathbf{u}_{T}\right\|_{H^{\frac{3}{2}(\Gamma, T(\Gamma))}}+\left\|\sigma_{\nu}(\mathbf{u})\right\|_{H^{\frac{1}{2}}(\Gamma)}\right)
$$

On montre qu'il existe une constante positive $C$ telle que:

$$
\begin{gathered}
\|\mathbf{u}\|_{\mathbb{H}^{2}(\Omega)}+\leq C\left\|\sigma_{S}(\mathbf{u})-\overline{\operatorname{div}_{T} \sigma_{T}^{0}}(\mathbf{u})\right\|_{H^{\frac{1}{2}}(\Gamma, T(\Gamma))}+ \\
C\left(\|\mathbf{u}-\operatorname{div} \sigma(\mathbf{u})\|_{\mathbb{L}^{2}(\Omega)}+\left\|\sigma_{\nu}(\mathbf{u})+\sigma_{T}^{0}(\mathbf{u}): \partial_{m} \nu\right\|_{H^{\frac{1}{2}}(\Gamma)}\right), \quad \forall \mathbf{u} \in \mathcal{Z}
\end{gathered}
$$

Si cela n'était pas vrai il existerait une suite $\left\{\mathbf{u}^{k}\right\} \subset \mathcal{Z}$ telle que:

$$
\left\{\begin{array}{l}
\left\|\mathbf{u}^{k}\right\|_{\mathbb{H}^{2}(\Omega)}=1, \quad \mathbf{u}^{k}-\overline{\operatorname{div} \sigma}\left(\mathbf{u}^{k}\right) \longrightarrow 0 \text { dans } \mathbb{L}^{2}(\Omega), \\
\sigma_{S}\left(\mathbf{u}^{k}\right)-\overline{\operatorname{div}_{T} \sigma_{T}^{0}}\left(\mathbf{u}^{k}\right) \longrightarrow 0 \text { dans } H^{\frac{1}{2}}(\Gamma, T(\Gamma)), \\
\sigma_{\nu}\left(\mathbf{u}^{k}\right)+\sigma_{T}^{0}\left(\mathbf{u}^{k}\right): \partial_{m} \nu \longrightarrow 0 \text { dans } H^{\frac{1}{2}}(\Gamma) .
\end{array}\right.
$$

En posant: $\mathbf{f}^{k}=\mathbf{u}^{k}-\operatorname{div} \sigma\left(\mathbf{u}^{k}\right), \mathbf{g}_{T}^{k}=\sigma_{S}\left(\mathbf{u}^{k}\right)-\overline{\operatorname{div}}_{T} \sigma_{T}^{0}\left(\mathbf{u}^{k}\right), g_{\nu}^{k}=$ $\sigma_{\nu}\left(\mathbf{u}^{k}\right)+\sigma_{T}^{0}\left(\mathbf{u}^{k}\right): \partial_{m} \nu$ et $\mathbf{g}^{k}=\mathbf{g}_{T}^{k}+g_{\nu}^{k} \nu$ on obtient:

$$
\left\{\begin{array}{c}
\left(\mathbf{u}^{k}, \mathbf{v}\right)_{\mathbb{L}^{2}(\Omega)}+\int_{\Omega} \sigma\left(\mathbf{u}^{k}\right): \epsilon(\mathbf{v}) d x+\int_{\Gamma} \sigma_{T}^{0}\left(\mathbf{u}^{k}\right): \epsilon_{T}^{0}(\mathbf{v}) d \Gamma= \\
\int_{\Omega} \mathbf{f}^{k} \mathbf{v} d x+\int_{\Gamma} \mathbf{g}^{k} \mathbf{v} d \Gamma, \quad \forall \mathbf{v} \in \mathbb{V}
\end{array}\right.
$$


$\left\{\mathbf{u}^{k}\right\}$ étant bornée dans $\mathbb{H}^{2}(\Omega)$ on a: $\mathbf{u}^{k} \rightarrow \mathbf{u} \in \mathbb{H}^{2}(\Omega)$ dans $\mathbb{H}^{2-s}(\Omega)$ fort, avec $0<s<\frac{1}{2}$. On peut alors passer à la limite dans (2.6); on obtient:

$$
(\mathbf{u}, \mathbf{v})_{\mathbb{L}^{2}(\Omega)}+\int_{\Omega} \sigma(\mathbf{u}): \epsilon(\mathbf{v}) d x+\int_{\Gamma} \sigma_{T}^{0}(\mathbf{u}): \epsilon_{T}^{0}(\mathbf{v}) d \Gamma=0, \quad \forall \mathbf{v} \in \mathbb{V}
$$

En prenant $\mathbf{v}=\mathbf{u}$ dans (2.7) on obtient $\mathbf{u}=0$; il vient alors de (2.5) $\operatorname{div} \sigma\left(\mathbf{u}^{k}\right) \rightarrow 0$ dans $\mathbb{L}^{2}(\Omega)$. De $\mathbf{u}^{k} \rightarrow 0$ dans $\mathbb{H}^{1}(\Omega)$ et $\operatorname{div} \sigma\left(\mathbf{u}^{k}\right) \rightarrow 0$ dans $\mathbb{L}^{2}(\Omega)$ le corollaire 2.1 donne: $\sigma\left(\mathbf{u}^{k}\right) . \nu=\sigma_{S}\left(\mathbf{u}^{k}\right)+\sigma_{\nu}\left(\mathbf{u}^{k}\right) \nu \rightarrow 0$ dans $\mathbb{H}^{-\frac{1}{2}}(\Gamma) ; \quad$ de $(2.5)$ on obtient alors $\overline{\operatorname{div}}_{T}^{0} \sigma_{T}^{0}\left(\mathbf{u}^{k}\right) \rightarrow 0$ dans $H^{-\frac{1}{2}}(\Gamma, T(\Gamma))$. Comme $\mathbf{u}_{\mid \Gamma}^{k} \rightarrow 0$ dans $\mathbb{H}^{\frac{3}{2}-s}(\Gamma), \quad\left(0<s<\frac{1}{2}\right)$, et $\sigma_{T}^{0}\left(\mathbf{u}^{k}\right)=\sigma_{T}^{0}\left(\mathbf{u}_{T}^{k}\right)+\sigma_{T}^{0}\left(u_{\nu}^{k} \nu\right)=\sigma_{T}^{0}\left(\mathbf{u}_{T}^{k}\right)+2 \mu u_{\nu} \partial_{m} \nu+\lambda^{*} u_{\nu} \operatorname{tr}\left(\partial_{m} \nu\right) I_{2}$ on obtient $\overline{\operatorname{div}}_{T} \sigma_{T}^{0}\left(\mathbf{u}_{T}^{k}\right) \rightarrow 0$ dans $H^{-\frac{1}{2}}(\Gamma, T(\Gamma))$ d'où $\mathbf{u}_{T}^{k} \rightarrow 0$ dans $H^{\frac{3}{2}}(\Gamma, T(\Gamma))$ d'après la proposition 2.2 .

De la dernière limite de $(2.5)$ on obtient alors $\sigma_{\nu}\left(\mathbf{u}^{k}\right) \rightarrow 0$ dans $H^{\frac{1}{2}}(\Gamma)$. La proposition 2.6 montre que $\mathbf{u}^{k} \rightarrow 0$ dans $\mathbb{H}^{2}(\Omega)$ ce qui contredit la première égalité de (2.5).

Soit $\mathbf{u} \in \mathcal{Z}$; on a $\partial_{\nu} \mathbf{u} \in \mathbb{H}^{\frac{1}{2}}(\Gamma)$; de (1.1)-(1.4) il vient

$\sigma_{S}(\mathbf{u}) \in H^{\frac{1}{2}}(\Gamma, T(\Gamma))$ d'où $\overline{\operatorname{div}_{T} \sigma_{T}^{0}}(\mathbf{u}) \in H^{\frac{1}{2}}(\Gamma, T(\Gamma))$.

Comme:

$\sigma_{T}^{0}(\mathbf{u})=\sigma_{T}^{0}\left(\mathbf{u}_{T}\right)+\sigma_{T}^{0}\left(u_{\nu} \nu\right)=\sigma_{T}^{0}\left(\mathbf{u}_{T}\right)+2 \mu\left(u_{\nu} \partial_{m} \nu\right)+\lambda^{*} u_{\nu} \operatorname{tr}\left(\partial_{m} \nu\right) I_{2}$ alors $\overline{\operatorname{div}_{T} \sigma_{T}^{0}}\left(\mathbf{u}_{T}\right) \in H^{\frac{1}{2}}(\Gamma, T(\Gamma))$; la proposition 2.2 montre que $\mathbf{u}_{T} \in H^{\frac{5}{2}}(\Gamma, T(\Gamma))$ et il existe une constante positive $C$ ne dépendant que de $\Omega$ telle que:

$$
\left\|\mathbf{u}_{T}\right\|_{H^{\frac{5}{2}(\Gamma, T(\Gamma))}} \leq C\left\|\mathbf{u}_{T}-\overline{\operatorname{div} \sigma_{T} \sigma_{T}^{0}}\left(\mathbf{u}_{T}\right)\right\|_{H^{\frac{1}{2}(\Gamma, T(\Gamma))}} .
$$


On a:

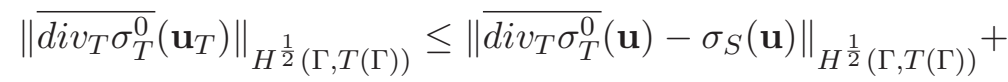

$$
\begin{aligned}
& \left\|\overline{\operatorname{div}_{T} \sigma_{T}^{0}}\left(u_{\nu} \partial_{m} \nu\right)\right\|_{H^{\frac{1}{2}(\Gamma, T(\Gamma))}}+\left\|\sigma_{S}(\mathbf{u})\right\|_{H^{\frac{1}{2}(\Gamma, T(\Gamma))}} \leq \\
& C\left(\|\mathbf{u}\|_{\mathbb{H}^{2}(\Omega)}+\left\|\overline{\operatorname{div}_{T} \sigma_{T}^{0}}(\mathbf{u})-\sigma_{S}(\mathbf{u})\right\|_{H^{\frac{1}{2}(\Gamma, T(\Gamma))}}\right) \leq \\
& C\left(\left\|\sigma_{S}(\mathbf{u})-\overline{\operatorname{div}_{T} \sigma_{T}^{0}}(\mathbf{u})\right\|_{H^{\frac{1}{2}}(\Gamma, T(\Gamma))}+\|\mathbf{u}-\operatorname{div} \sigma(\mathbf{u})\|_{\mathbb{L}^{2}(\Omega)}+\right. \\
& \left.\left\|\sigma_{\nu}(\mathbf{u})+\sigma_{T}^{0}(\mathbf{u}): \partial_{m} \nu\right\|_{H^{\frac{1}{2}(\Gamma)}}\right)
\end{aligned}
$$

Remarque 2.5. On a: $\mathcal{V}=D\left(\mathcal{A}^{\frac{1}{2}}\right)$ et $D\left(\mathcal{A}^{\frac{3}{2}}\right)=\{U \in \mathcal{V}: \mathcal{A} U \in \mathcal{V}\} ; S i$ $\left(\mathbf{u}_{1}, \mathbf{u}_{2}\right) \in D\left(\mathcal{A}^{\frac{3}{2}}\right)$ alors:

$\circ$ Les propositions 2.2 et 2.6 montrent que $\mathbf{u}_{1} \in \mathbb{H}^{2}(\Omega)$ et et il existe une constante positive $C$ qui ne dépend que de $\Omega$ telle que:

$$
\begin{gathered}
\left\|\mathbf{u}_{1}\right\|_{\mathbb{H}^{2}(\Omega)} \leq C\left(\left\|\mathbf{u}_{1}-\operatorname{div} \sigma\left(\mathbf{u}_{1}\right)\right\|_{\mathbb{L}^{2}(\Omega)}^{2}+\right. \\
\left.\left\|\mathbf{u}_{1 T}\right\|_{H^{\frac{3}{2}}(\Gamma, T(\Gamma))}^{2}+\left\|\sigma_{\nu}\left(\mathbf{u}_{1}\right)\right\|_{H^{\frac{1}{2}}(\Gamma)}^{2}\right)^{\frac{1}{2}}
\end{gathered}
$$

- La proposition 2.7 montre que $\mathbf{u}_{1} \in \mathbb{H}^{2}(\Omega), \mathbf{u}_{2 T} \in H^{\frac{5}{2}}(\Gamma, T(\Gamma))$ et et il existe une constante positive $C$ qui ne dépend que de $\Omega$ telle que:

$$
\begin{gathered}
\left\|\mathbf{u}_{1}\right\|_{\mathbb{H}^{2}(\Omega)}+\left\|\mathbf{u}_{2 T}\right\|_{H^{\frac{5}{2}(\Gamma, T(\Gamma))}} \leq \\
C\left\|\sigma_{S}\left(\mathbf{u}_{1}\right)-\overline{\operatorname{div} v_{T} \sigma_{T}^{0}}\left(\mathbf{u}_{2}\right)\right\|_{H^{\frac{1}{2}}(\Gamma, T(\Gamma))}+ \\
C\left(\left\|\mathbf{u}_{1}-\operatorname{div} \sigma\left(\mathbf{u}_{1}\right)\right\|_{\mathbb{L}^{2}(\Omega)}+\left\|\sigma_{\nu}\left(\mathbf{u}_{1}\right)+\sigma_{T}^{0}\left(\mathbf{u}_{2}\right): \partial_{m} \nu\right\|_{H^{\frac{1}{2}(\Gamma)}}\right) .
\end{gathered}
$$

Proposition 2.8. L'injection:

$$
\left\{\begin{array}{l}
D\left(\mathcal{A}^{\frac{3}{2}}\right) \hookrightarrow \mathbb{H}^{2}(\Omega) \times H^{\frac{5}{2}}(\Gamma, T(\Gamma)) \times H^{\frac{3}{2}}(\Gamma), \\
\left(v_{1}, v_{2}\right) \longrightarrow\left(v_{1}, v_{2 T}, v_{2 \nu}\right)
\end{array}\right.
$$

est continue.

Preuve. D'après la proposition 2.7 il suffit de montrer qu'il existe une constante positive $C$ telle que:

$$
\left\|v_{1}\right\|_{\mathbb{H}^{2}(\Omega)}+\left\|v_{2 T}\right\|_{H^{\frac{5}{2}}(\Gamma, T(\Gamma))} \leq C\|v\|_{D\left(\mathcal{A}^{\frac{3}{2}}\right)}, \quad \forall v=\left(v_{1}, v_{2}\right) \in D\left(\mathcal{A}^{\frac{3}{2}}\right)
$$


Dans le cas contraire on aura une suite $v^{k}=\left(v_{1}^{k}, v_{2}^{k}\right) \in D\left(\mathcal{A}^{\frac{3}{2}}\right)$ qui vérifie:

$$
\left\|v_{1}^{k}\right\|_{\mathbb{H}^{2}(\Omega)}+\left\|v_{2 T}^{k}\right\|_{H^{\frac{5}{2}}(\Gamma, T(\Gamma))}=1 \quad \text { et }\left\|v^{k}\right\|_{D\left(\mathcal{A}^{\frac{3}{2}}\right)} \longrightarrow 0
$$

De

$$
\mathcal{A} v^{k}=\left(\begin{array}{c}
-\operatorname{div} \sigma\left(v_{1}^{k}\right) \\
\sigma_{S}\left(v_{1}^{k}\right)-\overline{\operatorname{div}_{T} \sigma_{T}^{0}\left(v_{2}^{k}\right)} \\
\sigma_{\nu}\left(v_{1}^{k}\right)+\sigma_{T}^{0}\left(v_{2}^{k}\right): \partial_{m} \nu
\end{array}\right) \in \mathcal{V}
$$

il vient

$$
\begin{aligned}
& -\left(\operatorname{div} \sigma\left(v_{1}^{k}\right)_{\Gamma}\right)_{T}=\sigma_{S}\left(v_{1}^{k}\right)-\overline{\operatorname{div}_{T} \sigma_{T}^{0}\left(v_{2}^{k}\right)} \\
& -\bar{\nu} \operatorname{div} \sigma\left(v_{1}^{k}\right)_{\Gamma}=\sigma_{\nu}\left(v_{1}^{k}\right)+\sigma_{T}^{0}\left(v_{2}^{k}\right): \partial_{m} \nu
\end{aligned}
$$

et

$$
\begin{gathered}
\left\|v^{k}\right\|_{D\left(\mathcal{A}^{\frac{3}{2}}\right)}^{2}=\left\|v^{k}\right\|_{\mathcal{V}}^{2}+\left\|\mathcal{A} v^{k}\right\|_{\mathcal{V}}^{2}=\left\|v_{1}^{k}\right\|_{\mathbb{H}^{1}(\Omega)}^{2}+\left\|\operatorname{div} \sigma\left(v_{1}^{k}\right)\right\|_{\mathbb{H}^{1}(\Omega)}^{2}+ \\
\left\|v_{2 T}^{k}\right\|_{H^{1}(\Gamma, T(\Gamma))}^{2}+\left\|\sigma_{S}\left(v_{1}^{k}\right)-\overline{\operatorname{div}_{T} \sigma_{T}^{0}\left(v_{2}^{k}\right)}\right\|_{H^{1}(\Gamma, T(\Gamma))}^{2}
\end{gathered}
$$

La proposition 2.7 donne alors une contradiction:

\section{$3 \quad$ Existence et régularité des solutions}

Proposition 3.1. Soient $\mathbf{f} \in \mathbb{L}^{2}(\Omega)$ et $\mathbf{g}=\mathbf{g}_{T}+g_{\nu} \nu \in \mathbb{H}^{\frac{1}{2}}(\Gamma)$. Le problème:

$$
\left\{\begin{array}{l}
\mathbf{u}-\operatorname{div} \sigma(\mathbf{u})=\mathbf{f} \quad \text { dans } \quad \Omega \\
\sigma_{S}(\mathbf{u})-\overline{\operatorname{div}}_{T} \sigma_{T}^{0}(\mathbf{u})=\mathbf{g}_{T} \quad \text { sur } \quad \Gamma \\
\sigma_{\nu}(\mathbf{u})+\sigma_{T}^{0}(\mathbf{u}): \partial_{m} \nu=g_{\nu} \quad \text { sur } \quad \Gamma
\end{array}\right.
$$

admet une solution unique $\mathbf{u} \in \mathbb{V}$; de plus $\mathbf{u} \in \mathbb{H}^{2}(\Omega), \quad \mathbf{u}_{T} \in$ $H^{\frac{5}{2}}(\Gamma, T(\Gamma))$ et il existe une constante positive $C$ qui ne dépend que de $\Omega$ telle que:

$$
\|\mathbf{u}\|_{\mathbb{H}^{2}(\Omega)}+\left\|\mathbf{u}_{T}\right\|_{H^{\frac{5}{2}(\Gamma, T(\Gamma))}} \leq C\left(\|\mathbf{f}\|_{\mathbb{L}^{2}(\Omega)}+\|\mathbf{g}\|_{\mathbb{H}^{\frac{1}{2}}(\Gamma)}^{\frac{1}{2}}\right)
$$


Preuve. Le problème (3.1) a pour formulation variationnelle:

$$
\begin{gathered}
\int_{\Omega}(\mathbf{u v}+\sigma(\mathbf{u}): \epsilon(\mathbf{v})) d x+\int_{\Gamma} \sigma_{T}^{0}(\mathbf{u}): \epsilon_{T}^{0}(\mathbf{v}) d \Gamma= \\
\int_{\Omega} \mathbf{f} \mathbf{v} d x+\int_{\Gamma} \mathbf{g v} d \Gamma, \quad \forall \mathbf{v} \in \mathbb{V}
\end{gathered}
$$

D'après la proposition 2.3 ce problème admet une solution unique $\mathbf{u}$ dans $\mathbb{V}$; il suffit alors d'appliquer la proposition 2.7.

Proposition 3.2. Soient $\mathbf{f} \in \mathbb{L}^{2}(\Omega)$ et $\mathbf{g}=\mathbf{g}_{T}+g_{\nu} \nu \in \mathbb{L}^{2}(\Gamma)$. Le problème:

$$
\left\{\begin{array}{l}
\mathbf{u}-\operatorname{div} \sigma(\mathbf{u})=\mathbf{f} \quad \text { dans } \quad \Omega \\
\sigma_{S}(\mathbf{u})-\overline{\operatorname{div}}_{T} \sigma_{T}^{0}(\mathbf{u})=\mathbf{g}_{T} \quad \text { sur } \quad \Gamma \\
\sigma_{\nu}(\mathbf{u})+\sigma_{T}^{0}(\mathbf{u}): \partial_{m} \nu=g_{\nu} \quad \text { sur } \quad \Gamma
\end{array}\right.
$$

admet une solution unique $\mathbf{u} \in \mathbb{V}$; de plus $\mathbf{u} \in \mathbb{H}^{\frac{3}{2}}(\Omega), \mathbf{u}_{T} \in H^{2}(\Gamma, T(\Gamma))$, $\sigma(\mathbf{u}) . \nu \in \mathbb{L}^{2}(\Gamma)$ et il existe une constante positive $C$ qui ne dépend que de $\Omega$ telle que:

$$
\|\mathbf{u}\|_{\mathbb{H}^{\frac{3}{2}}(\Omega)}+\|\sigma(\mathbf{u}) \cdot \nu\|_{\mathbb{L}^{2}(\Gamma)}+\left\|\mathbf{u}_{T}\right\|_{H^{2}(\Gamma, T(\Gamma))} \leq C\left(\|\mathbf{f}\|_{\mathbb{L}^{2}(\Omega)}+\|\mathbf{g}\|_{\mathbb{L}^{2}(\Gamma)}\right)
$$

Preuve. On suppose que $\mathbf{f} \in \mathbb{V}^{\prime}$ et $\mathbf{g} \in \mathbb{H}^{-\frac{1}{2}}(\Gamma)$. Le problème (3.2) a pour formulation variationnelle:

$$
\begin{aligned}
\int_{\Omega}(\mathbf{u v}+\sigma(\mathbf{u}): \epsilon(\mathbf{v})) d x+\int_{\Gamma} \sigma_{T}^{0}(\mathbf{u}): \epsilon_{T}^{0}(\mathbf{v}) d \Gamma= \\
(\mathbf{f}, \mathbf{v})_{\mathbb{V}^{\prime}, \mathbb{V}}+\left(\mathbf{g}, \mathbf{v}_{\mid \Gamma}\right)_{\mathbb{H}^{-\frac{1}{2}}(\Gamma), \mathbb{H}^{\frac{1}{2}}(\Gamma)}, \quad \forall \mathbf{v} \in \mathbb{V}
\end{aligned}
$$

D'après la proposition 2.3 le problème (3.2) admet une solution (faible) unique $\mathbf{u}$ dans $\mathbb{V}$ et il existe une constante positive $C$ qui ne dépend que de $\Omega$ telle que:

$$
\|\mathbf{u}\|_{\mathbb{V}} \leq C\left(\|\mathbf{f}\|_{\mathbb{V}^{\prime}}+\|\mathbf{g}\|_{\mathbb{H}^{-\frac{1}{2}(\Gamma)}}\right)
$$

D'après le corollaire 2.1 on a $\sigma(\mathbf{u}) . \nu \in \mathbb{H}^{-\frac{1}{2}}(\Gamma)$ et

$$
\begin{gathered}
\|\sigma(\mathbf{u}) \cdot \nu\|_{\mathbb{H}^{-\frac{1}{2}}(\Gamma)}+\leq C\left(\|\mathbf{u}\|_{\mathbb{V}}+\|\operatorname{div} \sigma(\mathbf{u})\|_{\mathbb{L}^{2}(\Omega)}\right) \leq \\
C\left(\|\mathbf{f}\|_{\mathbb{L}^{2}(\Omega)}+\|\mathbf{g}\|_{\mathbb{H}^{-\frac{1}{2}}(\Gamma)}\right)
\end{gathered}
$$


L'application $(\mathbf{f}, \mathbf{g}) \rightarrow(\mathbf{u}, \sigma(\mathbf{u}) . \nu)$ est donc continue de $\mathbb{L}^{2}(\Omega) \times$ $\mathbb{H}^{-\frac{1}{2}}(\Gamma)$ dans $\mathbb{H}^{1}(\Omega) \times \mathbb{H}^{-\frac{1}{2}}(\Gamma)$.

D'autre part, d'après la proposition 3.1 , cette application est continue de $\mathbb{L}^{2}(\Omega) \times \mathbb{H}^{\frac{1}{2}}(\Gamma)$ dans $\mathbb{H}^{2}(\Omega) \times \mathbb{H}^{\frac{1}{2}}(\Gamma)$. On obtient, par interpolation, une application continue de $\mathbb{L}^{2}(\Omega) \times \mathbb{L}^{2}(\Gamma)$ dans $\mathbb{H}^{\frac{3}{2}}(\Omega) \times \mathbb{L}^{2}(\Gamma)$.

De (3.2) il vient $\overline{\operatorname{div}_{T} \sigma_{T}^{0}(\mathbf{u})} \in L^{2}(\Gamma, T(\Gamma))$ d'où $\overline{\operatorname{div}_{T} \sigma_{T}^{0}\left(\mathbf{u}_{T}\right)}=$ $\overline{\operatorname{div}_{T} \sigma_{T}^{0}(\mathbf{u})}-\overline{\operatorname{div}_{T}}\left(2 \mu u_{\nu} \partial_{m} \nu+\lambda^{*} u_{\nu} \operatorname{tr}\left(\partial_{m} \nu\right)\right) \in L^{2}(\Gamma, T(\Gamma))$ puisque $u_{\nu} \in$ $H^{1}(\Gamma)$; la proposition 2.2 donne alors $\mathbf{u}_{T} \in H^{2}(\Gamma, T(\Gamma))$ et

$$
\begin{gathered}
\left\|\mathbf{u}_{T}\right\|_{H^{2}(\Gamma, T(\Gamma))} \leq C\left\|\mathbf{u}_{T}-\overline{d i v_{T} \sigma_{T}^{0}}\left(\mathbf{u}_{T}\right)\right\|_{L^{2}(\Gamma, T(\Gamma))} \leq \\
C\left(\|\mathbf{u}\|_{\mathbb{H}^{\frac{3}{2}}(\Omega)}+\left\|\sigma_{S}(\mathbf{u})-\overline{d i v_{T} \sigma_{T}^{0}}(\mathbf{u})\right\|_{L^{2}(\Gamma, T(\Gamma))}+\left\|\sigma_{S}(\mathbf{u})\right\|_{L^{2}(\Gamma, T(\Gamma))}+\right. \\
\left.\left\|\overline{d i v_{T} \sigma_{T}^{0}}\left(u_{\nu} \nu\right)\right\|_{L^{2}(\Gamma, T(\Gamma))}\right) \leq C\left(\|\mathbf{f}\|_{\mathbb{L}^{2}(\Omega)}+\|\mathbf{g}\|_{\mathbb{L}^{2}(\Gamma)}\right)
\end{gathered}
$$

Proposition 3.3. Si $\left(\mathbf{u}_{1}, \mathbf{u}_{2}\right) \in D(\mathcal{A})$ alors $\mathbf{u}_{1} \in \mathbb{H}^{\frac{3}{2}}(\Omega), \quad \mathbf{u}_{2 T} \in$ $H^{2}(\Gamma, T(\Gamma)), \sigma(\mathbf{u}) . \nu \in \mathbb{L}^{2}(\Gamma)$ et l'application:

$$
\begin{aligned}
& D(\mathcal{A}) \longrightarrow \mathbb{H}^{\frac{3}{2}}(\Omega) \times H^{2}(\Gamma, T(\Gamma)) \times \mathbb{L}^{2}(\Gamma) \\
& \left(\mathbf{u}_{1}, \mathbf{u}_{2}\right) \longrightarrow\left(\mathbf{u}_{1}, \mathbf{u}_{2 T}, \sigma\left(\mathbf{u}_{1}\right) . \nu\right)
\end{aligned}
$$

est continue.

Preuve. On rappelle que $\left(\mathbf{u}_{1}, \mathbf{u}_{2}\right) \in D(\mathcal{A})$ si et seulement si $\left(\mathbf{u}_{1}, \mathbf{u}_{2}\right) \in$ $\mathcal{V}$ et

$$
\left\{\begin{array}{l}
\mathbf{u}_{1}-\operatorname{div} \sigma\left(\mathbf{u}_{1}\right) \in \mathbb{L}^{2}(\Omega) \\
\sigma_{S}\left(\mathbf{u}_{1}\right)-\overline{\operatorname{div}}_{T} \sigma_{T}^{0}\left(\mathbf{u}_{2}\right) \in L^{2}(\Gamma, T(\Gamma)) \\
\sigma_{\nu}\left(\mathbf{u}_{1}\right)+\sigma_{T}^{0}\left(\mathbf{u}_{2}\right): \partial_{m} \nu \in L^{2}(\Gamma)
\end{array}\right.
$$

La norme de $D(\mathcal{A})$ est définie par:

$$
\begin{gathered}
\left\|\left(\mathbf{u}_{1}, \mathbf{u}_{2}\right)\right\|_{D(\mathcal{A})}=\left(\left\|\mathbf{u}_{1}\right\|_{\mathbb{V}}^{2}+\left\|\operatorname{div} \sigma\left(\mathbf{u}_{1}\right)\right\|_{\mathbb{L}^{2}(\Omega)}^{2}+\right. \\
\left.\left\|\sigma_{S}\left(\mathbf{u}_{1}\right)-\overline{\operatorname{div}_{T} \sigma_{T}^{0}}\left(\mathbf{u}_{2}\right)\right\|_{L^{2}(\Gamma, T(\Gamma))}^{2}+\left\|\sigma_{\nu}\left(\mathbf{u}_{1}\right)+\sigma_{T}^{0}\left(\mathbf{u}_{2}\right): \partial_{m} \nu\right\|_{L^{2}(\Gamma)}^{2}\right)^{\frac{1}{2}}
\end{gathered}
$$

Il suffit alors d'appliquer la proposition 3.2.

On pose: $\quad \mathbf{u}=\mathbf{u}_{1}, \quad \mathbf{u}_{\mid \Gamma}=\mathbf{u}_{2}, \quad U=\left(\mathbf{u}_{1}, \mathbf{u}_{2}\right), \mathbf{g}=\mathbf{g}_{T}+g_{\nu} \nu$, $F=(\mathbf{f}, \mathbf{g}), U^{0}=\left(\mathbf{u}_{1}^{0}, \mathbf{u}_{2}^{0}\right)$ et $U^{1}=\left(\mathbf{u}_{1}^{1}, \mathbf{u}_{2}^{1}\right)$ alors le problème (1.5) s'écrit:

$$
U^{\prime \prime}+\mathcal{A} U=F, \quad U(0)=U^{0}, \quad U^{\prime}(0)=U^{1}
$$


Lemme 3.1. L'injection $\mathcal{V} \rightarrow \mathcal{H}$ est compacte à image dense.

Preuve. On commence par montrer la densité de $\mathcal{V}$ dans $\mathcal{H}$; Soit $\left(\mathbf{v}_{1}, \mathbf{v}_{2}\right) \in \mathcal{H}$; On considère une suite $\left\{\mathbf{w}_{2}^{k}\right\} \subset \mathcal{D}\left(\Gamma, \mathbb{R}^{3}\right)$ qui converge vers $\mathbf{v}_{2}$ dans $\mathbb{L}^{2}(\Gamma)$ et $\mathbf{u}_{2}^{k}$ la solution du problème:

$$
\left\{\begin{array}{l}
\mathbf{u}_{2}^{k}-\Delta \mathbf{u}_{2}^{k}=0 \text { dans } \Omega \\
\mathbf{u}_{2 \mid \Gamma}^{k}=\mathbf{w}_{2}^{k} \text { sur } \Gamma
\end{array}\right.
$$

alors $\mathbf{u}_{2}^{k} \rightarrow \mathbf{u}_{2}$ dans $\mathbb{L}^{2}(\Omega)$ solution du problème:

$$
\left\{\begin{array}{l}
\mathbf{u}_{2}-\Delta \mathbf{u}_{2}=0 \text { dans } \Omega \\
\mathbf{u}_{2 \mid \Gamma}=\mathbf{v}_{2} \text { sur } \Gamma
\end{array}\right.
$$

Soit $\left\{\mathbf{w}_{1}^{k}\right\}$ une suite de $\mathcal{D}\left(\Omega, \mathbb{R}^{3}\right)$ qui converge vers $\mathbf{v}_{1}-\mathbf{u}_{2}$ dans $\mathbb{L}^{2}(\Omega)$. On pose $\mathbf{v}_{1}^{k}=\mathbf{w}_{1}^{k}+\mathbf{u}_{2}^{k} ;\left(\mathbf{v}_{1}^{k}, \mathbf{v}_{1 \mid \Gamma}^{k}\right)$ est alors une suite de $\mathcal{V}$ qui converge vers $\left(\mathbf{v}_{1}, \mathbf{v}_{2}\right)$ dans $\mathcal{H}$.

La compacité de l'injection $\mathcal{V} \rightarrow \mathcal{H}$ est une conséquence immédiate de la compacité de l'injection $\mathbb{H}^{1}(\Omega) \rightarrow \mathbb{L}^{2}(\Omega)$. Le théorème (1.1) découle $\mathrm{du}$ théorème qui suit et de la proposition 3.3.

Theoreme 3.1. On donne: $s \geq 0$ un nombre réel, $F \in L^{1}\left(0, T ; D\left(\mathcal{A}^{s}\right)\right)$, $U^{0} \in D\left(\mathcal{A}^{s+\frac{1}{2}}\right)$ et $U^{1} \in D\left(\mathcal{A}^{s}\right)$. Le problème (3.4) admet une solution unique:

$U \in \mathcal{C}^{0}\left([0, T] ; D\left(\mathcal{A}^{s+\frac{1}{2}}\right)\right) \cap \mathcal{C}^{1}\left([0, T] ; D\left(\mathcal{A}^{s}\right)\right)$ et il existe une constante positive $C$ ne dépendant que de $\Omega$ telle que:

$$
\begin{aligned}
&\|U\|_{L^{\infty}\left(0, T ; D\left(\mathcal{A}^{s+\frac{1}{2}}\right)\right)}+\left\|U^{\prime}\right\|_{L^{\infty}\left(0, T ; D\left(\mathcal{A}^{s}\right)\right)} \leq \\
& C\left(\|F\|_{L^{1}\left(0, T ; D\left(\mathcal{A}^{s}\right)\right)}+\left\|U^{0}\right\|_{D\left(\mathcal{A}^{s+\frac{1}{2}}\right)}+\left\|U^{1}\right\|_{D\left(\mathcal{A}^{s}\right)}\right)
\end{aligned}
$$

Preuve. L'opérateur $\mathcal{A}$ est positif, auto-adjoint; d'après le lemme (3.1) l'injection: $\mathcal{V} \hookrightarrow \mathcal{H}$ est compacte à image dense; $D(\mathcal{A})$ est alors dense dans $\mathcal{V}$ et il existe dans $\mathcal{H}$ un système dénombrable de vecteurs propres de $\mathcal{A}$ complet dans $\mathcal{H}$ et dans $D(\mathcal{A})$. Si $\mathcal{A} U_{k}=\lambda_{k} U_{k}, k=1,2, \ldots$ alors:

$$
U \in D\left(\mathcal{A}^{s}\right) \Longleftrightarrow \sum_{k \geq 1} \lambda_{k}^{2 s}\left(U, U_{k}\right)_{\mathcal{H}}^{2}<+\infty
$$


où $\left((., .)_{\mathcal{H}}\right.$ est le produit scalaire de $\left.\mathcal{H}\right)$. Il suffit alors d'écrire explicitement la formule de résolution:

$$
\begin{gathered}
U(t)=\sum_{k=0}^{\infty} \cos \left(t \sqrt{\lambda_{k}}\right)\left(U^{0}, U_{k}\right)_{\mathcal{H}} U_{k}+\sum_{k=1}^{\infty} \sin \left(t \sqrt{\lambda_{k}}\right)\left(U^{1}, U_{k}\right) \mathcal{H} \frac{U_{k}}{\sqrt{\lambda_{k}}}+ \\
\sum_{k=1}^{\infty} \int_{0}^{t} \sin \left((t-s) \sqrt{\lambda_{k}}\right)\left(F(s), U_{k}\right)_{\mathcal{H}} \frac{U_{k}}{\sqrt{\lambda_{k}}} d s
\end{gathered}
$$

Remarque 3.1. Le problème (3.4) admet la formulation variationnelle:

$$
\left\{\begin{array}{l}
\left(U^{\prime \prime}, V\right)+a(U, V)=(F, V), \forall V=\left(\mathbf{v}_{1}, \mathbf{v}_{2}\right) \in \mathcal{V} \\
U(0)=U^{0}, \quad U^{\prime}(0)=U^{1}
\end{array}\right.
$$

avec:

$$
a(U, V)=\int_{\Omega} \sigma\left(\mathbf{u}_{1}\right): \epsilon\left(\mathbf{v}_{1}\right) d x+\int_{\Gamma} \sigma_{T}^{0}\left(\mathbf{u}_{2}\right): \epsilon_{T}^{0}\left(\mathbf{v}_{2}\right) d \Gamma
$$

Remarque 3.2. On suppose: $F \in L^{1}(0, T ; \mathcal{H}), U^{0} \in \mathcal{V}$ et $U^{1} \in \mathcal{H}$; l'énergie de la solution $U$ du problème (3.5) s'écrit:

$$
E(U, t)=\frac{1}{2}\left(a(U, U)+\left\|U^{\prime}\right\|_{\mathcal{H}}^{2}\right)
$$

\section{Théorème d'unicité}

\subsection{Une identité}

Proposition 4.1. Soient $\mathbf{q} \in \mathbb{W}^{1, \infty}(\bar{\Omega}),\left(\varphi^{0}, \varphi^{1}\right) \in D(\mathcal{A}) \times \mathcal{V}$ et $f=$ $\left(f_{1}, f_{2}\right) \in L^{1}(0, T ; \mathcal{V})$; la solution $\varphi=\left(\varphi_{1}, \varphi_{2}\right)$ du problème $(1.6)$ corre- 
spondant vérifie l'identité:

$$
\begin{gathered}
\left.\left(\varphi_{1}^{\prime},(\mathbf{q} \nabla) \varphi_{1}\right)_{\Omega}\right|_{0} ^{T}+\left.\left(\varphi_{2 T}^{\prime},\left(\pi \partial_{m} \varphi_{1 T} \pi+\varphi_{1 \nu}\left(\partial_{m} \nu\right)\right) \mathbf{q}_{T}\right)_{\Gamma}\right|_{0} ^{T}+ \\
\left(\varphi_{2 \nu}^{\prime},\left(-\overline{\varphi_{1 T}}\left(\partial_{m} \nu\right)\right) \mathbf{q}_{T}\right)_{\Gamma}||_{0}^{T}+\int_{Q_{T}} \sigma\left(\varphi_{1}\right): \nabla \mathbf{q} \nabla \varphi_{1} d x d t+= \\
\int_{Q_{T}} \frac{d i v \mathbf{q}}{2}\left(\left|\varphi_{1}^{\prime}\right|^{2}-\sigma\left(\varphi_{1}\right): \epsilon\left(\varphi_{1}\right)\right) d x d t-\int_{\Sigma_{T}} \frac{q_{\nu}}{2}\left(\mu\left|\partial_{\nu} \varphi_{1 T}\right|^{2}+\right. \\
\left.(2 \mu+\lambda)\left|\partial_{\nu} \varphi_{1 \nu}\right|^{2}\right) d \Sigma+\int_{\Sigma_{T}} \frac{q_{\nu}}{2}\left(2 \mu \epsilon_{T}^{0}\left(\varphi_{1}\right)+\lambda \operatorname{tr}\left(\epsilon_{T}^{0}\left(\varphi_{1}\right)\right) i_{2}\right): \epsilon_{T}^{0}\left(\varphi_{1}\right)+ \\
\left.\mu\left|\overline{\partial_{m} \varphi_{1 \nu}}-\left(\partial_{m} \nu\right) \varphi_{1 T}\right|^{2}-\left|\varphi_{1}^{\prime}\right|^{2}\right) d \Sigma+\int_{\Sigma_{T}} \frac{d i v_{T} \mathbf{q}_{T}}{2}\left(\left|\varphi_{2 T}^{\prime}\right|^{2}-\right. \\
\left.\sigma_{T}^{0}\left(\varphi_{1}\right): \epsilon_{T}^{0}\left(\varphi_{1}\right)\right) d \Sigma-\int_{\Sigma_{T}}\left(\sigma_{T}^{0}\left(\varphi_{2}\right): \partial_{m} \nu\right) \overline{\varphi_{1 T}}\left(\partial_{m} \nu\right) \mathbf{q}_{T} d \Sigma+ \\
\left.\int_{\Sigma_{T}} \sigma_{\Sigma_{T}}^{0}\left(\varphi_{2}\right):\left(\pi \partial_{m} \varphi_{2 T} \pi+\varphi_{2 \nu} \partial_{m} \nu\right) \pi \partial_{m} \mathbf{q}_{T} \pi\right) d \Sigma+ \\
\int_{\Sigma_{T}}\left(\sigma_{T}^{0}\left(\varphi_{2}\right)\left(\partial_{m} \nu\right) \mathbf{q}_{T}\right) d \Sigma-\int_{\Sigma_{T}} \sigma_{\nu}\left(\varphi_{1}\right)\left(\partial_{m} \varphi_{1 \nu}\right):\left(\mathbf{q}_{T}+\right. \\
\int_{\Sigma_{T}} \overline{f_{2 T}}\left(\pi \partial_{m} \varphi_{1 T} \pi+\varphi_{1 \nu} \partial_{m} \nu\right) \mathbf{q}_{T} d \Sigma-\int_{\Sigma_{T}} f_{2 \nu}\left(\bar{\varphi} \int_{1 T} \partial_{m} \nu\right) \mathbf{q}_{T} d \Sigma
\end{gathered}
$$

où $\mathcal{R}$ est le tenseur de courbure de $\Gamma,(c f .[6])$; c'est un tenseur une fois contravariant et trois fois covariant de composantes:

$$
\mathcal{R}_{\alpha \beta \gamma}^{\delta}=\Gamma_{\gamma \alpha, \beta}^{\delta}-\Gamma_{\beta \alpha, \gamma}^{\delta}+\Gamma_{\beta r}^{\delta} \Gamma_{\gamma \alpha}^{r}-\Gamma_{\gamma r}^{\delta} \Gamma_{\beta \alpha}^{r}, 1 \leq \alpha, \beta, \gamma, \delta \leq 2
$$

Les $\Gamma_{\lambda \mu}^{\nu}$ sont les symboles de Cristoffel, (cf.[1],[7],[12]).

Preuve. Les calculs qui suivent sont justifiés par le point (ii) du théorème 1.1. On multiplie:

La première équation de $(1.6)$ par $\left.(\mathbf{q} \nabla) \varphi_{1}\right)$ et on intègre sur $Q_{T}$; on obtient:

$$
\begin{gathered}
\left.\left(\varphi_{1}^{\prime},(\mathbf{q} \nabla) \varphi_{1}\right)_{\Omega}\right|_{0} ^{T}+\int_{Q_{T}} \frac{\operatorname{div} \mathbf{q}}{2}\left(\left|\varphi_{1}^{\prime}\right|^{2}-\sigma\left(\varphi_{1}\right): \epsilon\left(\varphi_{1}\right)\right) d x d t+ \\
\int_{Q_{T}}\left(\sigma\left(\varphi_{1}\right): \nabla \mathbf{q} \nabla \varphi_{1}\right) d \Sigma+\int_{\Sigma_{T}} \frac{q_{\nu}}{2}\left(\sigma\left(\varphi_{1}\right): \epsilon\left(\varphi_{1}\right)-\left|\varphi_{1}^{\prime}\right|^{2}\right) d \Sigma- \\
\left.\int_{\Sigma_{T}}\left(\sigma\left(\varphi_{1}\right) \cdot \nu\right)(\mathbf{q} \nabla) \varphi_{1} d x d t=\int_{Q_{T}} f_{1}(\mathbf{q} \nabla) \varphi_{1}\right) d x
\end{gathered}
$$


La deuxième équation de (1.6) $\operatorname{par}\left(\pi \partial_{m} \varphi_{1 T} \pi+\varphi_{1 \nu} \partial_{m} \nu\right) \mathbf{q}_{T}$ et on intègre sur $\Sigma_{T}$; on obtient:

$$
\begin{aligned}
& \left.\left(\varphi_{2 T}^{\prime},\left(\pi \partial_{m} \varphi_{1 T} \pi+\varphi_{1 \nu} \partial_{m} \nu\right) \mathbf{q}_{T}\right)_{\Gamma}\right|_{0} ^{T}- \\
& \int_{\Sigma_{T}} \varphi_{2 T}^{\prime}\left(\pi \partial_{m} \varphi_{1 T}^{\prime} \pi+\varphi_{1 \nu}^{\prime} \partial_{m} \nu\right) \mathbf{q}_{T} d \Sigma+ \\
& \int_{\Sigma_{T}} \sigma_{T}^{0}\left(\varphi_{2}\right): \pi \partial_{m}\left(\left(\pi \partial_{m} \varphi_{1 T} \pi+\varphi_{1 \nu}\left(\partial_{m} \nu\right)\right) \mathbf{q}_{T}\right) d \Sigma+ \\
& \int_{\Sigma_{T}} \overline{\sigma_{S}\left(\varphi_{1}\right)}\left(\pi \partial_{m} \varphi_{1 T} \pi+\varphi_{1 \nu} \partial_{m} \nu\right) \mathbf{q}_{T} d \Sigma= \\
& \int_{\Sigma_{T}} \overline{f_{2 T}}\left(\pi \partial_{m} \varphi_{1 T} \pi+\varphi_{1 \nu} \partial_{m} \nu\right) \mathbf{q}_{T} d \Sigma
\end{aligned}
$$

La troisième équation de (1.6) par $\left(-\overline{\varphi_{1 T}} \partial_{m} \nu\right) \mathbf{q}_{T}$ et on intègre sur $\Sigma_{T}$; on obtient:

$$
\begin{aligned}
& \left.\left(\varphi_{2 \nu}^{\prime},\left(-\overline{\varphi_{1 T}} \partial_{m} \nu\right) \mathbf{q}_{T}\right)\right|_{\Gamma} ^{T}+\int_{\Sigma_{T}} \varphi_{2 \nu}^{\prime}\left(\overline{\varphi_{1 T}^{\prime}} \partial_{m} \nu\right) \mathbf{q}_{T} d \Sigma- \\
& \int_{\Sigma_{T}}\left(\sigma_{T}^{0}\left(\varphi_{2}\right): \partial_{m} \nu\right)\left(\overline{\varphi_{1 T}} \partial_{m} \nu\right) \mathbf{q}_{T} d \Sigma- \\
& \int_{\Sigma_{T}} \sigma_{\nu}\left(\varphi_{1}\right)\left(\overline{\varphi_{1 T}} \partial_{m} \nu\right) \mathbf{q}_{T} d \Sigma=-\int_{\Sigma_{T}} f_{2 \nu}\left(\overline{\varphi_{1 T}} \partial_{m} \nu\right) \mathbf{q}_{T} d \Sigma
\end{aligned}
$$

La somme des trois égalités précédentes donne:

$$
\begin{aligned}
& \left.\left(\varphi_{1}^{\prime},(\mathbf{q} \nabla) \varphi_{1}\right)_{\Omega}\right|_{0} ^{T}+\left.\left(\varphi_{2 T}^{\prime},\left(\pi \partial_{m} \varphi_{1 T} \pi+\varphi_{1 \nu} \partial_{m} \nu\right) \mathbf{q}_{T}\right)_{\Gamma}\right|_{0} ^{T}+ \\
& \left.\left(\varphi_{2 \nu}^{\prime},\left(-\overline{\varphi_{1 T}} \partial_{m} \nu\right) \mathbf{q}_{T}\right)_{\Gamma}\right|_{0} ^{T}+\int_{Q_{T}} \frac{\operatorname{div} \mathbf{q}}{2}\left(\left|\varphi_{1}^{\prime}\right|^{2}-\sigma\left(\varphi_{1}\right): \epsilon\left(\varphi_{1}\right)\right) d x d t+ \\
& \int_{Q_{T}} \sigma\left(\varphi_{1}\right): \nabla \mathbf{q} \nabla \varphi_{1} d x d t+\int_{\Sigma_{T}}^{Q_{T}} \frac{q_{\nu}}{2}\left(\sigma\left(\varphi_{1}\right): \epsilon\left(\varphi_{1}\right)-\left|\varphi_{1}^{\prime}\right|^{2}\right) d \Sigma- \\
& \int_{\Sigma_{T}}^{Q_{T}}\left(\sigma\left(\varphi_{1}\right) \cdot \nu\right)(\mathbf{q} \nabla) \varphi_{1} d \Sigma-\int_{\Sigma_{T}}^{\Sigma_{T}} \varphi_{2 T}^{\prime}\left(\pi \partial_{m} \varphi_{1 T}^{\prime} \pi+\varphi_{1 \nu}^{\prime} \partial_{m} \nu\right) \mathbf{q}_{T} d \Sigma- \\
& \int_{\Sigma_{T}}^{\Sigma_{T}} \varphi_{2 \nu}^{\prime}\left(-\overline{\varphi_{1 T}^{\prime}} \partial_{m} \nu\right) \mathbf{q}_{T} d \Sigma+\int_{\Sigma_{T}}^{\sigma_{S}\left(\varphi_{1}\right)}\left(\pi \partial_{m} \varphi_{1 T} \pi+\varphi_{1 \nu} \partial_{m} \nu\right) \mathbf{q}_{T} d \Sigma+ \\
& \int_{\Sigma_{T}}^{\Sigma_{T}} \sigma_{\nu}\left(\varphi_{1}\right)\left(-\overline{\varphi_{1 T}} \partial_{m} \nu\right) \mathbf{q}_{T} d \Sigma+\int_{\Sigma_{T}}\left(\sigma_{T}^{0}\left(\varphi_{2}\right): \partial_{m} \nu\right)\left(-\overline{\varphi_{1 T}} \partial_{m} \nu\right) \mathbf{q}_{T} d \Sigma+ \\
& \int_{\Sigma_{T}}^{T} \sigma_{T}^{0}\left(\varphi_{2}\right): \pi \partial_{m}\left(\left(\pi \partial_{m} \varphi_{1 T} \pi+\varphi_{1 \nu}\left(\partial_{m} \nu\right)\right) \mathbf{q}_{T}\right) d \Sigma= \\
& \left.\int_{Q_{T}}^{\sum_{T}} f_{1}(\mathbf{q} \nabla) \varphi_{1}\right) d x+ \\
& \int_{\Sigma_{T}}^{Q_{T}} \overline{f_{2 T}}\left(\pi \partial_{m} \varphi_{1 T} \pi+\varphi_{1 \nu} \partial_{m} \nu\right) \mathbf{q}_{T} d \Sigma+\int_{\Sigma_{T}} f_{2 \nu}\left(-\overline{\varphi_{1 T}} \partial_{m} \nu\right) \mathbf{q}_{T} d \Sigma
\end{aligned}
$$


On calcule ensuite les différents termes.

$$
\begin{gathered}
\int_{\Sigma_{T}} \overline{\varphi_{2 T}^{\prime}}\left(\pi \partial_{m} \varphi_{2 T}^{\prime} \pi\right) \mathbf{q}_{T} d \Sigma= \\
\frac{1}{2} \int_{\Sigma_{T}} \partial_{m}\left(\left|\varphi_{2 T}^{\prime}\right|^{2}\right) \mathbf{q}_{T} d \Sigma=-\int_{\Sigma_{T}} \frac{\operatorname{div}_{T} \mathbf{q}_{T}}{2}\left|\varphi_{2 T}^{\prime}\right|^{2} d \Sigma
\end{gathered}
$$

Sachant que $(\mathbf{q} \nabla) \varphi_{1}=\overline{\nabla \varphi_{1}} \cdot \mathbf{q}$, on obtient de (2.1):

$$
\begin{aligned}
& \left(\sigma\left(\varphi_{1}\right) . \nu\right)(\mathbf{q} \nabla) \varphi_{1}=\overline{\sigma_{S}\left(\varphi_{1}\right)}\left(\pi \partial_{m} \varphi_{1 T} \pi+\varphi_{1 \nu} \partial_{m} \nu\right) \mathbf{q}_{T}+ \\
& \sigma_{\nu}\left(\varphi_{1}\right)\left(\partial_{m} \varphi_{1 \nu}-\overline{\varphi_{1 T}} \partial_{m} \nu\right) \mathbf{q}_{T}+q_{\nu}\left(\sigma\left(\varphi_{1}\right) . \nu\right) \partial_{\nu} \varphi_{1}
\end{aligned}
$$

Enfin, un calcul trop long pour être donné ici, montre que:

$$
\begin{aligned}
& \sigma_{T}^{0}\left(\varphi_{2}\right): \pi \partial_{m}\left(\left(\pi \partial_{m} \varphi_{1 T} \pi+\varphi_{1 \nu}\left(\partial_{m} \nu\right)\right) \mathbf{q}_{T}\right) \pi= \\
& \sigma_{T}^{0}\left(\varphi_{2}\right):\left(\pi\left(\partial_{m} \varphi_{2 T}\right) \pi+\varphi_{2 \nu}\left(\partial_{m} \nu\right)\right)\left(\pi \partial_{m} \mathbf{q}_{T} \pi\right)+ \\
& \left(\partial_{m} \varphi_{2 \nu}\right)\left(\sigma_{T}^{0}\left(\varphi_{2}\right)\left(\partial_{m} \nu\right) \mathbf{q}_{T}\right)+\frac{1}{2} \partial_{m}\left(\sigma_{T}^{0}\left(\varphi_{2}\right): \epsilon_{T}^{0}\left(\varphi_{2}\right)\right) \mathbf{q}_{T}- \\
& \left(\sigma_{T}^{0}\left(\varphi_{2}\right): \partial_{m} \nu\right)\left(\partial_{m} \varphi_{1 \nu}\right) \mathbf{q}_{T}+\left(\mathcal{R}: \sigma_{T}^{0}\left(\varphi_{1}\right)\right):\left(\varphi_{1 T} \bigotimes \mathbf{q}_{T}\right)
\end{aligned}
$$

En remplaçant les termes précédents par leurs valeurs on obtient:

$$
\begin{aligned}
& \left.\left(\varphi_{1}^{\prime},(\mathbf{q} \nabla) \varphi_{1}\right)_{\Omega}\right|_{0} ^{T}+\left.\left(\varphi_{2 T}^{\prime},\left(\pi \partial_{m} \varphi_{1 T} \pi+\varphi_{1 \nu}\left(\partial_{m} \nu\right)\right) \mathbf{q}_{T}\right)_{\Gamma}\right|_{0} ^{T}- \\
& \left.\left(\varphi_{2 \nu}^{\prime},\left(\overline{\varphi_{1 T}} \partial_{m} \nu\right) \mathbf{q}_{T}\right) \Gamma\right|_{0} ^{T}+\int_{Q_{T}} \sigma\left(\varphi_{1}\right): \nabla \mathbf{q} \nabla \varphi_{1} d x d t+ \\
& \int_{Q_{T}} \frac{\operatorname{div} \mathbf{q}}{2}\left(\left|\varphi_{1}^{\prime}\right|^{2}-\sigma\left(\varphi_{1}\right): \epsilon\left(\varphi_{1}\right)\right) d x d t+ \\
& \int_{\Sigma_{T}}^{\Sigma_{m}}\left(\partial_{m} \varphi_{2 \nu}\right)\left(\sigma_{T}^{0}\left(\varphi_{2}\right)\left(\partial_{m} \nu\right) \mathbf{q}_{T}\right) d \Sigma+ \\
& \int_{\Sigma_{T}}^{\Sigma_{T}} \frac{q_{\nu}}{2}\left(\sigma\left(\varphi_{1}\right): \epsilon\left(\varphi_{1}\right)-2\left(\sigma\left(\varphi_{1}\right) \nu\right)\left(\partial_{\nu} \varphi_{1}\right)-\left|\varphi_{1}^{\prime}\right|^{2}\right) d \Sigma- \\
& \int_{\Sigma_{T}}^{\Sigma_{T}}\left(\sigma_{T}^{0}\left(\varphi_{2}\right): \partial_{m} \nu\right) \overline{\varphi_{1 T}}\left(\partial_{m} \nu\right) \mathbf{q}_{T} d \Sigma-\int_{\Sigma_{T}} \sigma_{\nu}\left(\varphi_{1}\right)\left(\partial_{m} \varphi_{1 \nu}\right) \mathbf{q}_{T} d \Sigma+ \\
& \int_{\Sigma_{T}}^{\Sigma_{T}} \frac{\operatorname{div}_{T} \mathbf{q}_{T}}{2}\left(\left|\varphi_{2 T}^{\prime}\right|^{2}-\sigma_{T}^{0}\left(\varphi_{1}\right): \epsilon_{T}^{0}\left(\varphi_{1}\right)\right) d \Sigma+ \\
& \int_{\Sigma_{T}}^{\Sigma_{T}}\left(\sigma_{T}^{0}\left(\varphi_{2}\right):\left(\pi \partial_{m} \varphi_{2 T} \pi+\varphi_{2 \nu} \partial_{m} \nu\right)\left(\pi \partial_{m} \mathbf{q}_{T} \pi\right)\right) d \Sigma+ \\
& \int_{\Sigma_{T}}^{\Sigma_{T}}\left(\mathcal{R}: \sigma_{T}^{0}\left(\varphi_{1}\right)\right):\left(\varphi_{1 T} \bigotimes \mathbf{q}_{T}\right) d \Sigma=\int_{Q_{T}} f_{1}(\mathbf{q} \nabla) \varphi_{1} d x+ \\
& \int_{\Sigma_{T}}^{\Sigma_{T}} \overline{f_{2 T}}\left(\pi \partial_{m} \varphi_{1 T} \pi+\varphi_{1 \nu} \partial_{m} \nu\right) \mathbf{q}_{T} d \Sigma-\int_{\Sigma_{T}}^{Q_{T}} f_{2 \nu}\left(\overline{\varphi_{1 T}} \partial_{m} \nu\right) \mathbf{q}_{T} d \Sigma
\end{aligned}
$$


Les relations (1.1)-(1.4) et la remarque 2.1 donnent:

$$
\begin{aligned}
& \sigma\left(\varphi_{1}\right): \epsilon\left(\varphi_{1}\right)-2\left(\sigma\left(\varphi_{1}\right) \nu\right)\left(\partial_{\nu} \varphi_{1}\right)=\sigma_{T}\left(\varphi_{1}\right): \epsilon_{T}\left(\varphi_{1}\right)+ \\
& 2 \overline{\sigma_{S}\left(\varphi_{1}\right)} \epsilon_{S}\left(\varphi_{1}\right)-2 \overline{\sigma_{S}\left(\varphi_{1}\right)} \partial_{\nu} \varphi_{1 T}-\sigma_{\nu}\left(\varphi_{1}\right) \epsilon_{\nu}\left(\varphi_{1}\right)= \\
& \left(2 \mu \epsilon_{T}\left(\varphi_{1}\right)+\lambda \operatorname{tr}\left(\epsilon_{T}\left(\varphi_{1}\right)\right) I_{2}\right): \epsilon_{T}\left(\varphi_{1}\right)+\lambda \epsilon_{\nu}\left(\varphi_{1}\right) \operatorname{tr}\left(\epsilon_{T}\left(\varphi_{1}\right)\right)+ \\
& 2 \overline{\sigma_{S}\left(\varphi_{1}\right)}\left(\epsilon_{S}\left(\varphi_{1}\right)-\partial_{\nu} \varphi_{1 T}\right)-\sigma_{\nu}\left(\varphi_{1}\right) \epsilon_{\nu}\left(\varphi_{1}\right)= \\
& \left(2 \mu \epsilon_{T}^{0}\left(\varphi_{1}\right)+\lambda \operatorname{tr}\left(\epsilon_{T}^{0}\left(\varphi_{1}\right)\right) I_{2}\right): \epsilon_{T}^{0}\left(\varphi_{1}\right)+ \\
& \mu\left(\left|\overline{\partial_{m} \varphi_{1 \nu}}-\left(\partial_{m} \nu\right) \varphi_{1 T}\right|^{2}-\left|\partial_{\nu} \varphi_{1 T}\right|^{2}\right)-(2 \mu+\lambda)\left|\partial_{\nu} \varphi_{1 \nu}\right|^{2}
\end{aligned}
$$

D'où:

$$
\begin{aligned}
& \int_{\Sigma_{T}}\left(\sigma\left(\varphi_{1}\right): \epsilon\left(\varphi_{1}\right)-2\left(\sigma\left(\varphi_{1}\right) \nu\right)\left(\partial_{\nu} \varphi_{1}\right)\right) d \Sigma= \\
& \int_{\Sigma_{T}}\left(\left(2 \mu \epsilon_{T}^{0}\left(\varphi_{1}\right)+\lambda \operatorname{tr}\left(\epsilon_{T}^{0}\left(\varphi_{1}\right)\right) i_{2}\right): \epsilon_{T}^{0}\left(\varphi_{1}\right)+\right. \\
& \left.\mu\left|\frac{\partial_{m} \varphi_{1 \nu}}{\partial_{m}}-\left(\partial_{m} \nu\right) \varphi_{1 T}\right|^{2}-\mu\left|\partial_{\nu} \varphi_{1 T}\right|^{2}-(2 \mu+\lambda)\left|\partial_{\nu} \varphi_{1 \nu}\right|^{2}\right) d \Sigma
\end{aligned}
$$

On obtient (4.1) à partir de (4.3) et (4.4).

Lemme 4.1. Pour tout champ scalaire $\eta$ défini sur $\Gamma$ et tout champ tangent $\mathbf{v}_{T}$ supposés réguliers on a:

$$
\left(\partial_{m} \eta\right) \mathbf{v}_{T}=\operatorname{tr}\left(\mathbf{v}_{T} \partial_{m} \eta\right) \quad \text { et } \quad \operatorname{div}_{T} \mathbf{v}_{T}=\operatorname{tr}\left(\pi \partial_{m} \mathbf{v}_{T} \pi\right)
$$

Preuve. $\quad$ Si $\mathbf{v}_{T}=v^{\alpha} \mathbf{a}_{\alpha}$ alors $\operatorname{tr}\left(\mathbf{v}_{T} \partial_{m} \eta\right)=\mathbf{a}^{\alpha}\left(\mathbf{v}_{T} \partial_{m} \eta\right) \mathbf{a}_{\alpha}=v^{\alpha} \partial_{\alpha} \eta$. D'autre part $\left(\partial_{m} \eta\right) \mathbf{v}_{T}=\left(\partial_{\beta} \eta\right) \mathbf{a}^{\beta}\left(v^{\alpha} \mathbf{a}_{\alpha}\right)=\left(\partial_{\alpha} \eta\right) v^{\alpha}$, ce qui montre la première relation.

Soit $\psi \in \mathcal{D}(\Gamma)$ un champ scalaire; par définition on a, (d'après la première relation du lemme) :

$$
\int_{\Gamma} \psi d i v_{T} \mathbf{v}_{T} d \Gamma=-\int_{\Gamma}\left(\partial_{m} \psi\right) \mathbf{v}_{T} d \Gamma=-\int_{\Gamma} \operatorname{tr}\left(\mathbf{v}_{T}\left(\partial_{m} \psi\right)\right) d \Gamma
$$

Or $\pi \partial_{m}\left(\psi \mathbf{v}_{T}\right) \pi=\mathbf{v}_{T} \partial_{m} \psi+\psi \pi\left(\partial_{m} \mathbf{v}_{T}\right) \pi$; d'où:

$$
\int_{\Gamma} \psi d i v_{T} \mathbf{v}_{T} d \Gamma=-\int_{\Gamma} \operatorname{tr}\left(\pi \partial_{m}\left(\psi \mathbf{v}_{T}\right) \pi\right) d \Gamma+\int_{\Gamma} \psi \operatorname{tr}\left(\pi\left(\partial_{m} \mathbf{v}_{T}\right) \pi\right) d \Gamma
$$

Si $\tau_{T}$ est un champ régulier d'endomorphismes du plan tangent à $\Gamma$ et $\mathbf{v}_{T}$ un champ tangent régulier on a par définition:

$$
\int_{\Gamma} d i v_{T} \tau_{T} \mathbf{v}_{T} d \Gamma=-\int_{\Gamma} \operatorname{tr}\left(\tau_{T} \pi\left(\partial_{m} \mathbf{v}_{T}\right) \pi\right) d \Gamma
$$


d'où:

$$
\int_{\Gamma} \psi \operatorname{div}_{T} \mathbf{v}_{T} d \Gamma=\int_{\Gamma} \psi\left(\operatorname{div}_{T} I_{2}\right) \mathbf{v}_{T} d \Gamma+\int_{\Gamma} \psi \operatorname{tr}\left(\pi\left(\partial_{m} \mathbf{v}_{T}\right) \pi\right) d \Gamma
$$

On a pour tout $\mathbf{w}_{T} \in \mathcal{D}(\Gamma, T(\Gamma))$

$$
\int_{\Gamma}\left(\operatorname{div}_{T} I_{2}\right) \mathbf{w}_{T} d \Gamma=-\int_{\Gamma} \operatorname{tr}\left(\pi\left(\partial_{m} \mathbf{w}_{T}\right) \pi\right) d \Gamma=-\int_{\Gamma} \operatorname{div}_{T} \mathbf{w}_{T} d \Gamma=0,
$$

par définition de $\operatorname{div}_{T} \mathbf{w}_{T}$, d'où $\operatorname{div}_{T} I_{2}=0$, ce qui termine la démonstration.

Lemme 4.2. Pour $\mathbf{q}=\mathbf{x}-\mathbf{x}_{0}=\mathbf{q}_{T}+q_{\nu}$ o on $a$

$$
\left\{\begin{array}{l}
\pi \partial_{m} \mathbf{q}_{T} \pi+q_{\nu} \partial_{m} \nu=I_{2} \\
\nabla_{T} q_{\nu}=\left(\partial_{m} \nu\right) \mathbf{q}_{T} \\
\operatorname{div}_{T} \mathbf{q}_{T}=2-q_{\nu} \operatorname{tr}\left(\partial_{m} \nu\right)
\end{array}\right.
$$

Preuve. On a, (cf.[1],[7],[12]):

$$
\nabla \mathbf{q}=I_{3} \quad \text { et } \quad \nabla \mathbf{q}_{\mid \Gamma}=\left(\begin{array}{cc}
\pi \overline{\partial_{m} \mathbf{q}_{T}} \pi+q_{\nu} \partial_{m} \nu, & \overline{\partial_{m} q_{\nu}}-\left(\partial_{m} \nu\right) \mathbf{q}_{T} \\
\overline{\partial_{\nu} \mathbf{q}_{T}} & \partial_{\nu} q_{\nu}
\end{array}\right)
$$

Ce qui donne: $\pi \overline{\partial_{m} \mathbf{q}_{T}} \pi+q_{\nu} \partial_{m} \nu=I_{2}$ et $\overline{\partial_{m} q_{\nu}}-\left(\partial_{m} \nu\right) \mathbf{q}_{T}=0$; la première relation de (4.5) est obtenue par transposition, sachant que l'opérateur de courbure $\partial_{m} \nu$ est symétrique; la troisième relation découle du lemme 4.1 et de la première relation de (4.5).

Proposition 4.2. Soient $\mathbf{q}=\mathbf{x}-\mathbf{x}_{0}, \varphi^{0}=\left(\varphi_{1}^{0}, \varphi_{2}^{0}\right) \in D(\mathcal{A})$ et $\varphi^{1}=$ $\left(\varphi_{1}^{1}, \varphi_{2}^{1}\right) \in \mathcal{V}$. La solution $\varphi=\left(\varphi_{1}, \varphi_{2}\right)$ du problème homogène (1.6) 
vérifie l'identité:

$$
\begin{gathered}
T E_{0}+\left.\left(\varphi_{1}^{\prime},(\mathbf{q} \nabla) \varphi_{1}+\varphi_{1}\right)_{\Omega}\right|_{0} ^{T}+\left(\varphi_{2 T}^{\prime},\left(\pi \partial_{m} \varphi_{1 T} \pi\right.\right. \\
\left.\left.+\varphi_{1 \nu}\left(\partial_{m} \nu\right)\right) \mathbf{q}_{T}+\varphi_{2 T}\right)\left._{\Gamma}\right|_{0} ^{T}+\left.\left(\varphi_{2 \nu}^{\prime},\left(-\overline{\varphi_{1 T}}\left(\partial_{m} \nu\right)\right) \mathbf{q}_{T}+\varphi_{2 \nu}\right)_{\Gamma}\right|_{0} ^{T}= \\
\quad \frac{1}{2} \int_{\Sigma_{T}}\left(\left|\varphi_{2 T}^{\prime}\right|^{2}+3\left|\varphi_{2 \nu}^{\prime}\right|^{2}+\sigma_{T}^{0}\left(\varphi_{2}\right): \epsilon_{T}^{0}\left(\varphi_{2}\right)\right) d \Sigma \\
\int_{\Sigma_{T}} \frac{q_{\nu}}{2}\left(\left|\varphi_{2}^{\prime}\right|^{2}+\mu\left(\left|\partial_{\nu} \varphi_{1 T}\right|^{2}-\left|\overline{\partial_{m} \varphi_{1 \nu}}-\left(\partial_{m} \nu\right) \varphi_{1 T}\right|^{2}\right)-\left(2 \mu \epsilon_{T}^{0}\left(\varphi_{1}\right)+\right.\right. \\
\left.\left.\lambda \operatorname{tr}\left(\epsilon_{T}^{0}\left(\varphi_{1}\right)\right) i_{2}\right): \epsilon_{T}^{0}\left(\varphi_{1}\right)\right) d \Sigma+ \\
\quad \int_{\Sigma_{T}} \frac{q_{\nu}}{2}\left(\left(\left|\varphi_{2 T}^{\prime}\right|^{2}-\sigma_{T}^{0}\left(\varphi_{2}\right): \epsilon_{T}^{0}\left(\varphi_{2}\right)\right) \operatorname{tr}\left(\partial_{m} \nu\right)+\right. \\
\quad 2\left(\sigma_{T}^{0}\left(\varphi_{1}\right):\left(\pi \partial_{m} \varphi_{1 T} \pi+\varphi_{1 \nu} \partial_{m} \nu\right) \partial_{m} \nu+(2 \mu+\lambda)\left|\partial_{\nu} \varphi_{1 \nu}\right|^{2}\right) d \Sigma+ \\
\int_{\Sigma_{T}}\left(\sigma_{T}^{0}\left(\varphi_{2}\right): \partial_{m} \nu\right) \overline{\varphi_{1 T}}\left(\partial_{m} \nu\right) \mathbf{q}_{T} d \Sigma-\int_{\Sigma_{T}} \partial_{m} \varphi_{1 \nu}\left(\sigma_{T}^{0}\left(\varphi_{1}\right)\left(\partial_{m} \nu\right) \mathbf{q}_{T}\right) d \Sigma+ \\
\int_{\Sigma_{T}} \sigma_{\nu}\left(\varphi_{1}\right)\left(\partial_{m} \varphi_{1 \nu}\right) \mathbf{q}_{T} d \Sigma-\int_{\Sigma_{T}}\left(\mathcal{R}: \sigma_{T}^{0}\left(\varphi_{1}\right)\right):\left(\varphi_{1 T} \bigotimes \mathbf{q}_{T}\right) d \Sigma .
\end{gathered}
$$

Preuve. Il suffit de montrer l'identité (4.6) pour $\left(\varphi^{0}, \varphi^{1}\right) \in D\left(\mathcal{A}^{\frac{3}{2}}\right) \times$ $D(\mathcal{A})$ et de généraliser par un argument de densité. On commence par exprimer la conservation de l'énergie:

$$
\begin{gathered}
E_{0}=\frac{1}{2} \int_{\Omega}\left(\sigma\left(\varphi_{1}\right): \epsilon\left(\varphi_{1}\right)+\left|\varphi_{1}^{\prime}\right|^{2}\right) d \Omega+ \\
\quad \frac{1}{2} \int_{\Gamma}\left(\sigma_{T}^{0}\left(\varphi_{2}\right): \epsilon_{T}^{0}\left(\varphi_{2}\right)+\left|\varphi_{2}^{\prime}\right|^{2}\right) d \Gamma
\end{gathered}
$$

D'après la remarque $3.1 \varphi$ vérifie: $\left(\varphi^{\prime \prime}, v\right)+a(\varphi, v)=0, \forall v \in \mathcal{V}$; en prenant $v=\varphi$ on obtient: $\partial_{t}\left(\varphi^{\prime}, \varphi\right)_{\mathcal{H}}-\left\|\varphi^{\prime}\right\|_{\mathcal{H}}^{2}+a(\varphi, \varphi)=0$, d'où la relation d'équipartition de l'énergie:

$$
\left.\left(\varphi^{\prime}, \varphi\right)_{\mathcal{H}}\right|_{0} ^{T}=\int_{0}^{T}\left(\left|\varphi^{\prime}\right|_{\mathcal{H}}^{2}-a(\varphi, \varphi)\right) d t
$$

On obtient (4.6) à partir de (4.1) en tenant compte de (4.5), (4.7) et (4.8). $\bar{\Omega}\}$.

Soit $\mathbf{x}_{0} \in \mathbb{R}^{3}$; on pose: $\mathbf{q}(\mathbf{x})=\mathbf{x}-\mathbf{x}_{0}$ et $R\left(\mathbf{x}_{0}\right)=\operatorname{Max}\left\{\left|\mathbf{x}-\mathbf{x}_{0}\right| ; x \in\right.$ 
Proposition 4.3. Soient $\varphi^{0}=\left(\varphi_{1}^{0}, \varphi_{2}^{0}\right) \in D(\mathcal{A})$ et $\varphi^{1}=\left(\varphi_{1}^{1}, \varphi_{2}^{1}\right) \in$ $\mathcal{V}$. Il existe trois constantes positives $\delta, \gamma$ et $c_{2}$, qui ne dépendent que de $\Omega$ telles que si $T_{0}=2 R\left(\mathbf{x}_{0}\right)(\sqrt{\delta}+\sqrt{\gamma})$ et $M=\operatorname{Max}\{(1+$ $\left.\left.\gamma R\left(\mathbf{x}_{0}\right)\right) \frac{1}{\sqrt{\gamma}}, 2\left(R\left(\mathbf{x}_{0}\right) \sqrt{\delta}\right)^{-1}\right)$ alors l'énergie $E_{0}$ de la solution $\varphi=\left(\varphi_{1}, \varphi_{2}\right)$ du problème homogène (1.6) vérifie l'identité:

$$
\begin{gathered}
\left(T-T_{0}\right) E_{0}+\frac{1}{R\left(\mathbf{x}_{0}\right) \sqrt{\gamma}}\left|\varphi_{1}^{0}\right|_{\Omega}^{2}+\frac{1}{2 R\left(\mathbf{x}_{0}\right) \sqrt{\delta}}\left|\varphi_{1}^{0}\right|_{\Gamma}^{2} \leq \\
\frac{1}{2} \int_{\Sigma_{T}}\left(\left|\varphi_{2 T}^{\prime}\right|^{2}+3\left|\varphi_{2 \nu}^{\prime}\right|^{2}\right) d \Sigma+ \\
\int_{\Sigma_{T}} \frac{q_{\nu}}{2}\left(\left|\varphi_{2}^{\prime}\right|^{2}+\mu\left(\left|\partial_{\nu} \varphi_{1 T}\right|^{2}-\left|\overline{\partial_{m} \varphi_{1 \nu}}-\left(\partial_{m} \nu\right) \varphi_{1 T}\right|^{2}\right) d \Sigma+\right. \\
\int_{\Sigma_{T}} \frac{q_{\nu}}{2}\left((2 \mu+\lambda)\left|\partial_{\nu} \varphi_{1 \nu}\right|^{2}-\left(2 \mu \sigma_{T}^{0}\left(\varphi_{1}\right)+\lambda \operatorname{tr}\left(\epsilon_{T}^{0}\left(\varphi_{1}\right)\right) I_{2}\right): \epsilon_{T}^{0}\left(\varphi_{1}\right)\right) d \Sigma+ \\
M c_{2} \int_{\Sigma_{T}}\left(\left|\varphi_{1}\right|^{2}+\left|\varphi_{1}^{\prime}\right|^{2}\right) d \Sigma+\int_{\Sigma_{T}} \sigma_{\nu}\left(\varphi_{1}\right)\left(\partial_{m} \varphi_{1 \nu}\right) \mathbf{q}_{T}+ \\
\int_{\Sigma_{T}} \frac{q_{\nu}}{2}\left(\left(\left|\varphi_{2 T}^{\prime}\right|^{2}-\sigma_{T}^{0}\left(\varphi_{2}\right): \epsilon_{T}^{0}\left(\varphi_{2}\right)\right) \operatorname{tr}\left(\partial_{m} \nu\right)+\right. \\
\left.2 \sigma_{T}^{0}\left(\varphi_{1}\right):\left(\pi \partial_{m} \varphi_{1 T} \pi+\varphi_{1 \nu} \partial_{m} \nu\right) \partial_{m} \nu\right) d \Sigma+ \\
\int_{\Sigma_{T}}\left(\sigma_{T}^{0}\left(\varphi_{2}\right): \partial_{m} \nu\right) \overline{\varphi_{1 T}}\left(\partial_{m} \nu\right) \mathbf{q}_{T} d \Sigma- \\
\partial_{\Sigma_{T}}\left(\sigma_{T}^{0}\left(\varphi_{1}\right)\left(\partial_{m} \nu\right) \mathbf{q}_{T}\right) d \Sigma-\int_{\Sigma_{T}}\left(\mathcal{R}: \sigma_{T}^{0}\left(\varphi_{1}\right)\right):\left(\varphi_{1 T} \bigotimes \mathbf{q}_{T}\right) d \Sigma
\end{gathered}
$$

Preuve. On majore les termes intégrés de la relation (4.6) par la méthode de V. KOMORNIK (cf.[5],[9]). On pose:

$$
\xi(t)=\left(\varphi_{1}^{\prime}(t),(\mathbf{q} \nabla) \varphi_{1}(t)+\varphi_{1}(t)\right)_{\Omega}=\left(\varphi_{1}^{\prime}(t),\left(q_{k} \partial_{k} \varphi_{1}(t)+\varphi_{1}(t)\right)_{\Omega}\right.
$$

( $\partial_{k}$ désigne la dérivée par rapport à la variable d'espace $x_{k}$ ).

On a:

$$
\left|\left(\varphi_{1}^{\prime},(\mathbf{q} \nabla) \varphi_{1}+\varphi_{1}\right)_{\Omega}\right|_{0}^{T}|\leq| \xi(0)|+| \xi(T) \mid
$$

On écrit:

$$
|\xi(t)| \leq \frac{\alpha}{2}\left|\varphi_{1}^{\prime}(t)\right|_{\Omega}^{2}+\frac{1}{2 \alpha}\left|q_{k} \partial_{k} \varphi_{1}(t)+\varphi_{1}(t)\right|_{\Omega}^{2}
$$


où $\alpha$ est une constante positive qui sera choisie plus loin. On a:

$$
\begin{gathered}
\left|q_{k} \partial_{k} \varphi_{1}(t)+\varphi_{1}(t)\right|_{\Omega}^{2}=\left|q_{k} \partial_{k} \varphi_{1}(t)\right|_{\Omega}^{2}+\left|\varphi_{1}(t)\right|_{\Omega}^{2}+\int_{\Omega} q_{k} \partial_{k}\left(\left|\varphi_{1}(t)\right|^{2}\right) d x= \\
\left|q_{k} \partial_{k} \varphi_{1}(t)\right|_{\Omega}^{2}-2\left|\varphi_{1}(t)\right|_{\Omega}^{2}+\int_{\Gamma} q_{\nu}\left|\varphi_{1}(t)\right|^{2} d \Gamma \leq \\
R\left(\mathbf{x}_{0}\right)^{2}\left|\nabla \varphi_{1}(t)\right|_{\Omega}^{2}-2\left|\varphi_{1}(t)\right|_{\Omega}^{2}+R\left(\mathbf{x}_{0}\right) \int_{\Gamma}\left|\varphi_{1}(t)\right|^{2} d \Gamma .
\end{gathered}
$$

Sachant, (cf.[9]p.231), qu'il existe $\gamma>0$ tel que:

$$
\int_{\Omega}|\nabla v|^{2} d x \leq \gamma\left(\int_{\Omega} \sigma(v): \epsilon(v) d x+\int_{\Gamma}|v|^{2} d \Gamma\right), \forall v \in \mathbb{H}^{1}(\Omega) .
$$

on obtient:

$$
\begin{gathered}
\left|q_{k} \partial_{k} \varphi_{1}(t)+\varphi_{1}(t)\right|_{\Omega}^{2} \leq \gamma R\left(\mathbf{x}_{0}\right)^{2} \int_{\Omega} \sigma\left(\varphi_{1}\right): \epsilon\left(\varphi_{1}\right) d x-2\left|\varphi_{1}(t)\right|_{\Omega}^{2}+ \\
\left(R\left(\mathbf{x}_{0}\right)+\gamma R\left(\mathbf{x}_{0}\right)^{2}\right) \int_{\Gamma}\left|\varphi_{1}(t)\right|^{2} d \Gamma
\end{gathered}
$$

d'où:

$$
\begin{aligned}
|\xi(t)| \leq & \frac{\alpha}{2}\left|\varphi_{1}(t)\right|_{\Omega}^{2}+\frac{\gamma R\left(\mathbf{x}_{0}\right)^{2}}{2 \alpha} \int_{\Omega} \sigma\left(\varphi_{1}\right): \epsilon\left(\varphi_{1}\right) d x- \\
& \frac{1}{\alpha}\left|\varphi_{1}(t)\right|_{\Omega}^{2}+\frac{1}{2 \alpha}\left(R\left(\mathbf{x}_{0}\right)+\gamma R\left(\mathbf{x}_{0}\right)^{2}\right) \int_{\Gamma}\left|\varphi_{1}(t)\right|^{2} d \Gamma
\end{aligned}
$$

Le choix $\alpha=R\left(\mathbf{x}_{0}\right) \sqrt{\gamma}$ donne:

$$
\begin{aligned}
&|\xi(t)| \leq R\left(\mathbf{x}_{0}\right) \sqrt{\gamma}\left(\frac{1}{2}\left|\varphi_{1}^{\prime}(t)\right|_{\Omega}^{2}+\frac{1}{2} \int_{\Omega} \sigma\left(\varphi_{1}\right): \epsilon\left(\varphi_{1}\right) d x\right)- \\
& \frac{1}{R\left(\mathbf{x}_{0}\right) \sqrt{\gamma}}\left|\varphi_{1}(t)\right|_{\Omega}^{2}+\frac{1}{2 \sqrt{\gamma}}\left(1+\gamma R\left(\mathbf{x}_{0}\right)\right) \int_{\Gamma}\left|\varphi_{1}(t)\right|^{2} d \Gamma \leq \\
& R\left(\mathbf{x}_{0}\right) \sqrt{\gamma} E_{0}-\frac{R\left(\mathbf{x}_{0}\right) \sqrt{\gamma}}{2} \int_{\Gamma} \sigma_{T}^{0}\left(\varphi_{1}(t)\right): \epsilon_{T}^{0}\left(\varphi_{1}(t)\right) d \Gamma- \\
& \frac{1}{R\left(\mathbf{x}_{0}\right) \sqrt{\gamma}}\left|\varphi_{1}(t)\right|_{\Omega}^{2}+\frac{1}{2 \sqrt{\gamma}}\left(1+\gamma R\left(\mathbf{x}_{0}\right)\right) \int_{\Gamma}\left|\varphi_{1}(t)\right|^{2} d \Gamma .
\end{aligned}
$$

$\varphi_{1 \mid \Gamma}$ est un élément de $H^{1}\left(0, T ; \mathbb{L}^{2}(\Gamma)\right)$ qui s'injecte continument dans $C^{0}\left([0, T] ; \mathbb{L}^{2}(\Gamma)\right)$; il existe alors une constante positive $c_{1}$ telle que:

$$
\left\|\varphi_{1}\right\|_{L^{\infty}\left(0, T ; \mathbb{L}^{2}(\Gamma)\right)}^{2} \leq c_{1}\left(\left\|\varphi_{1}\right\|_{L^{2}\left(0, T ; \mathbb{L}^{2}(\Gamma)\right)}^{2}+\left\|\varphi_{1}^{\prime}\right\|_{L^{2}\left(0, T ; \mathbb{L}^{2}(\Gamma)\right)}^{2}\right)
$$


On obtient:

$$
\begin{aligned}
& |\xi(t)| \leq R\left(\mathbf{x}_{0}\right) \sqrt{\gamma} E_{0}-\frac{R\left(\mathbf{x}_{0}\right) \sqrt{\gamma}}{2} \int_{\Gamma} \sigma_{T}^{0}\left(\varphi_{1}(t)\right): \epsilon_{T}^{0}\left(\varphi_{1}(t)\right) d \Gamma- \\
& \quad \frac{1}{R\left(\mathbf{x}_{0}\right) \sqrt{\gamma}}\left|\varphi_{1}(t)\right|_{\Omega}^{2}+\frac{c_{1}}{2 \sqrt{\gamma}}\left(1+\gamma R\left(\mathbf{x}_{0}\right)\right) \int_{\Sigma_{T}}\left(\left|\varphi_{1}(t)\right|^{2}+\left|\varphi_{1}^{\prime}(t)\right|^{2}\right) d \Gamma .
\end{aligned}
$$

et

$$
\begin{gathered}
\left|\left(\varphi_{1}^{\prime},(\mathbf{q} \nabla) \varphi_{1}+\varphi_{1}\right)_{\Omega}\right|_{0}^{T} \leq 2 R\left(\mathbf{x}_{0}\right) \sqrt{\gamma} E_{0}-\frac{1}{R\left(\mathbf{x}_{0}\right) \sqrt{\gamma}}\left|\varphi_{1}^{0}\right|_{\Omega}^{2}+ \\
\left(1+\gamma R\left(\mathbf{x}_{0}\right)\right) \frac{c_{1}}{\sqrt{\gamma}} \int_{\Sigma_{T}}\left(\left|\varphi_{1}(t)\right|^{2}+\left|\varphi_{1}^{\prime}(t)\right|^{2}\right) d \Sigma
\end{gathered}
$$

Pour majorer l'intégrale sur $\Gamma$ on pose: $\mathcal{Y}=\mathcal{Y}_{T}+\mathcal{Y}_{\nu} \nu$ avec:

$$
\mathcal{Y}_{T}=\left(\pi \partial_{m} \varphi_{1 T} \pi+\varphi_{1 \nu} \partial_{m} \nu\right) \mathbf{q}_{T} \text { et } \mathcal{Y}_{\nu}=-\overline{\varphi_{1 T}}\left(\partial_{m} \nu\right) \mathbf{q}_{T}
$$

Soit $\eta(t)=\left(\varphi_{2}^{\prime}, \mathcal{Y}+\varphi_{2}\right)_{\Gamma}(t)$. On écrit:

$$
|\eta(t)| \leq \frac{\beta}{2}\left|\varphi_{2}^{\prime}(t)\right|_{\Gamma}^{2}+\frac{1}{2 \beta}\left|\mathcal{Y}+\varphi_{2}(t)\right|_{\Gamma}^{2}
$$

où $\beta$ est une constante positive qui sera choisie plus loin. On a:

$$
\begin{aligned}
& \left|\mathcal{Y}+\varphi_{2}(t)\right|_{\Gamma}^{2}=\left|\left(\pi \partial_{m} \varphi_{1 T} \pi+\varphi_{1 \nu} \partial_{m} \nu\right) \mathbf{q}_{T}\right|_{\Gamma}^{2}+\left|\overline{\varphi_{1 T}}\left(\partial_{m} \nu\right) \mathbf{q}_{T}\right|_{\Gamma}^{2}+ \\
& \left|\varphi_{2}\right|_{\Gamma}^{2}+2 \int_{\Gamma} \overline{\varphi_{1 T}}\left(\pi \partial_{m} \varphi_{1 T} \pi+\varphi_{1 \nu} \partial_{m} \nu\right) \mathbf{q}_{T} d \Gamma-2 \int_{\Gamma} \varphi_{2 \nu}\left(\overline{\varphi_{1 T}} \partial_{m} \nu\right) \mathbf{q}_{T} d \Gamma \\
& =\left|\left(\pi \partial_{m} \varphi_{1 T} \pi+\varphi_{1 \nu} \partial_{m} \nu\right) \mathbf{q}_{T}\right|_{\Gamma}^{2}+\left|\left(\overline{\varphi_{1 T}} \partial_{m} \nu\right) \mathbf{q}_{T}\right|_{\Gamma}^{2}+\left|\varphi_{2}\right|_{\Gamma}^{2}+ \\
& \int_{\Gamma} \partial_{m}\left(\left|\varphi_{1 T}\right|^{2}\right) \mathbf{q}_{T} d \Gamma=\left|\left(\pi \partial_{m} \varphi_{1 T} \pi+\varphi_{1 \nu} \partial_{m} \nu\right) \mathbf{q}_{T}\right|_{\Gamma}^{2}+\left|\left(\overline{\varphi_{1 T}} \partial_{m} \nu\right) \mathbf{q}_{T}\right|_{\Gamma}^{2}- \\
& \quad\left|\varphi_{2}\right|_{\Gamma}^{2}+\int_{\Gamma} q_{\nu}\left|\varphi_{1 T}\right|^{2} \operatorname{tr}\left(\partial_{m} \nu\right) d \Gamma+2\left|\varphi_{2 \nu}\right|_{\Gamma}^{2}
\end{aligned}
$$

Alors:

$$
\begin{gathered}
|\eta(t)| \leq \frac{\beta}{2}\left|\varphi_{2}^{\prime}(t)\right|_{\Gamma}^{2}+\frac{1}{2 \beta}\left|\left(\pi \partial_{m} \varphi_{1 T} \pi+\varphi_{1 \nu} \partial_{m} \nu\right) \mathbf{q}_{T}\right|_{\Gamma}^{2}+\frac{1}{\beta}\left|\varphi_{2 \nu}\right|_{\Gamma}^{2}+ \\
\frac{1}{2 \beta}\left|\overline{\varphi_{1 T}}\left(\partial_{m} \nu\right) \mathbf{q}_{T}\right|_{\Gamma}^{2}-\frac{1}{2 \beta}\left|\varphi_{2}\right|_{\Gamma}^{2}+\frac{1}{2 \beta} \int_{\Gamma} q_{\nu}\left|\varphi_{1 T}\right|^{2} \operatorname{tr}\left(\partial_{m} \nu\right) d \Gamma
\end{gathered}
$$

D'après la proposition (2.4), il existe $\delta>0$ tel que:

$$
\begin{gathered}
\left|\left(\pi \partial_{m} \varphi_{1 T} \pi+\varphi_{1 \nu} \partial_{m} \nu\right) \mathbf{q}_{T}\right|_{\Gamma}^{2}+\left|\overline{\varphi_{1 T}}\left(\partial_{m} \nu\right) \mathbf{q}_{T}\right|_{\Gamma}^{2}+\int_{\Gamma} q_{\nu}\left|\varphi_{1 T}\right|^{2} \operatorname{tr}\left(\partial_{m} \nu\right) d \Gamma \\
\quad \leq \delta R\left(\mathbf{x}_{0}\right)^{2}\left(\int_{\Omega} \sigma\left(\varphi_{1}\right): \epsilon\left(\varphi_{1}\right) d x+\int_{\Gamma}\left(\sigma_{T}^{0}\left(\varphi_{1}\right): \epsilon_{T}^{0}\left(\varphi_{1}\right)+\left|\varphi_{1}\right|^{2}\right) d \Gamma\right)
\end{gathered}
$$


Alors:

$$
\begin{aligned}
& |\eta(t)| \leq \frac{\beta}{2}\left|\varphi_{2}^{\prime}(t)\right|_{\Gamma}^{2}+\frac{\delta R\left(\mathbf{x}_{0}\right)^{2}}{2 \beta}\left(\int_{\Omega} \sigma\left(\varphi_{1}\right): \epsilon\left(\varphi_{1}\right) d x+\right. \\
& \quad\left(\int_{\Gamma}\left(\sigma_{T}^{0}\left(\varphi_{1}\right): \epsilon_{T}^{0}\left(\varphi_{1}\right)+\left|\varphi_{1}\right|^{2}\right) d \Gamma\right)-\frac{1}{2 \beta}\left|\varphi_{2}(t)\right|_{\Gamma}^{2}+\frac{1}{\beta}\left|\varphi_{2 \nu}(t)\right|_{\Gamma}^{2}
\end{aligned}
$$

Le choix $\beta=R\left(\mathbf{x}_{0}\right) \sqrt{\delta}$ et la compacité de l'injection

$$
H^{1}\left(0, T ; L^{2}(\Gamma)\right) \rightarrow \mathcal{C}\left([0, T] ; L^{2}(\Gamma)\right)
$$

donnent:

$$
|\eta(t)| \leq R\left(\mathbf{x}_{0}\right) \sqrt{\delta} E_{0}-\frac{1}{2 R\left(\mathbf{x}_{0}\right) \sqrt{\delta}}\left|\varphi_{1}(t)\right|_{\Gamma}^{2}+\frac{c_{1}}{R\left(\mathbf{x}_{0}\right) \sqrt{\delta}} \int_{\Sigma_{T}}\left(\left|\varphi_{1}\right|^{2}+\left|\varphi_{1}^{\prime}\right|^{2}\right) d \Sigma
$$

et

$$
\begin{gathered}
\left|\left(\varphi_{2}^{\prime}, \mathcal{Y}+\varphi_{2}\right)_{\Gamma}\right|_{0}^{T} \leq 2 R\left(\mathbf{x}_{0}\right) \sqrt{\delta} E_{0}-\frac{1}{2 R\left(\mathbf{x}_{0}\right) \sqrt{\delta}}\left|\varphi_{1}^{0}\right|_{\Gamma}^{2}+ \\
\frac{2 c_{1}}{R\left(\mathbf{x}_{0}\right) \sqrt{\delta}} \int_{\Sigma_{T}}\left(\left|\varphi_{1}\right|^{2}+\left|\varphi_{1}^{\prime}\right|^{2}\right) d \Sigma
\end{gathered}
$$

Les estimations (4.10) et (4.11) montrent que les termes intégrés sont majorés par:

$$
\begin{gathered}
2 R\left(\mathbf{x}_{0}\right)(\sqrt{\gamma}+\sqrt{\delta}) E_{0}-\frac{1}{R\left(\mathbf{x}_{0}\right) \sqrt{\gamma}}\left|\varphi_{1}^{0}\right|_{\Omega}^{2}-\frac{1}{2 R\left(\mathbf{x}_{0}\right) \sqrt{\delta}}\left|\varphi_{1}^{0}\right|_{\Gamma}^{2}+ \\
M c_{2} \int_{\Sigma_{T}}\left(\left|\varphi_{1}\right|^{2}+\left|\varphi_{1}^{\prime}\right|^{2}\right) d \Sigma
\end{gathered}
$$

Ce qui permet d'obtenir l'estimation (4.9).

\section{démonstration du théorème 1.2}

Preuve. On utilise l'estimation (4.9). On a $\overline{\partial_{m} \varphi_{1 \nu}}=\nabla_{T} \varphi_{1 \nu}$. De (1.1)- (1.4) on obtient:

$$
\partial_{\nu} \varphi_{1 T}=\mu^{-1} \sigma_{S}\left(\varphi_{1}\right)+\left(\partial_{m} \nu\right) \varphi_{1 T}-\nabla_{T} \varphi_{1 \nu}
$$

et

$$
\partial_{\nu} \varphi_{1 \nu}=(2 \mu+\lambda)^{-1}\left(\sigma_{\nu}\left(\varphi_{1}\right)-\lambda \operatorname{tr}\left(\epsilon_{T}^{0}\left(\varphi_{1}\right)\right) .\right.
$$


La proposition 2.1 donne alors:

$$
\begin{aligned}
& \int_{\Sigma_{T}}\left|\partial_{\nu} \varphi_{1}\right|^{2} d \Sigma \leq C \int_{\Sigma_{T}}\left(\left|\nabla_{T} \varphi_{1 \nu}\right|^{2}+\right. \\
& \left.\left|\varphi_{1}\right|^{2}+\sigma_{T}^{0}\left(\varphi_{1}\right): \epsilon_{T}^{0}\left(\varphi_{1}\right)+\left|\sigma\left(\varphi_{1}\right) \cdot \nu\right|^{2}\right) d \Sigma
\end{aligned}
$$

où $C$ est une constante positive qui ne dépend que de $\Omega$.

De $\sigma_{T}^{0}\left(\varphi_{1}\right)=2 \mu \epsilon_{T}^{0}\left(\varphi_{1}\right)+\lambda^{*} \operatorname{tr}\left(\epsilon_{T}^{0}\left(\varphi_{1}\right)\right) I_{2}$ on obtient, grâce à la proposition 2.1

$$
\begin{gathered}
\left|\int_{\Sigma_{T}} \partial_{m} \varphi_{1 \nu}\left(\sigma_{T}^{0}\left(\varphi_{1}\right)\left(\partial_{m} \nu\right) \mathbf{q}_{T}\right) d \Sigma\right| \leq C\left(\left\|\nabla_{T} \varphi_{1 \nu}\right\|_{L^{2}\left(0, T ; L^{2}(\Gamma, T(\Gamma))\right.}^{2}+\right. \\
\left.\left\|\varphi_{1 \nu}\right\|_{L^{2}\left(0, T ; L^{2}(\Gamma)\right)}^{2}+\left\|\varphi_{1 T}\right\|_{L^{2}\left(0, T ; H^{1}(\Gamma, T(\Gamma))\right.}^{2}\right) \leq \\
C \int_{\Sigma_{T}}\left(\left|\nabla_{T} \varphi_{1 \nu}\right|^{2}+\left|\varphi_{1}\right|^{2}+\sigma_{T}^{0}\left(\varphi_{1}\right): \epsilon_{T}^{0}\left(\varphi_{1}\right)\right) d \Sigma
\end{gathered}
$$

Les autres termes de (4.9) sont estimés de façon analogue en appliquant la proposition 2.1 .

\section{Contrôlabilité}

\subsection{Régularité des solutions faibles}

Proposition 5.1. Soit $f=\partial_{t} g, g \in L^{1}(0, T ; D(\mathcal{A}))$; alors le problème:

$$
\theta^{\prime \prime}+\mathcal{A} \theta=f, \quad \theta(0)=\theta^{\prime}(0)=0
$$

admet une solution unique: $\theta \in \mathcal{C}^{0}([0, T] ; D(\mathcal{A}))$. $\theta^{\prime}(T)$ est défini comme élément de $\mathcal{V}$ et il existe une constante $C>0$ telle que:

$$
\|\theta\|_{L^{\infty}(0, T ; D(\mathcal{A}))}+\left\|\theta^{\prime}(T)\right\|_{\mathcal{V}} \leq C\|g\|_{L^{1}(0, T ; D(\mathcal{A}))}
$$

Preuve. On prend $g \in \mathcal{D}(0, T ; D(\mathcal{A}))$ puis on généralise par densité. Soient $\alpha$ et $\beta$ définies par:

$$
\begin{aligned}
& \alpha^{\prime \prime}+\mathcal{A} \alpha=g, \quad \alpha(0)=\alpha^{\prime}(0)=0 \quad \text { et } \\
& \beta^{\prime \prime}+\mathcal{A} \beta=\mathcal{A} g, \quad \beta(0)=\beta^{\prime}(0)=0 .
\end{aligned}
$$


Alors $\alpha \in \mathcal{C}^{0}\left([0, T] ; D\left(\mathcal{A}^{\frac{3}{2}}\right)\right) \cap \mathcal{C}^{1}([0, T] ; D(\mathcal{A}))$ et $\beta \in \mathcal{C}^{0}([0, T] ; \mathcal{V}) \cap$ $\mathcal{C}^{1}([0, T] ; \mathcal{H})$ et on a:

$$
\begin{gathered}
\|\alpha\|_{L^{\infty}\left(0, T ; D\left(\mathcal{A}^{\frac{3}{2}}\right)\right)}+\left\|\alpha^{\prime}\right\|_{L^{\infty}(0, T ; D(\mathcal{A}))} \leq C\|g\|_{L^{1}(0, T ; D(\mathcal{A}))} \\
\|\beta\|_{L^{\infty}(0, T ; \mathcal{V})}+\left\|\beta^{\prime}\right\|_{L^{\infty}(0, T ; \mathcal{H})} \leq C\|\mathcal{A} g\|_{L^{1}(0, T ; \mathcal{H})} \leq C\|g\|_{L^{1}(0, T ; D(\mathcal{A}))}
\end{gathered}
$$

or $\theta=\alpha^{\prime}$ d'où: $\theta \in \mathcal{C}^{0}([0, T] ; D(\mathcal{A}))$ et $\|\theta\|_{L^{\infty}(0, T ; D(\mathcal{A}))} \leq$

$C\|g\|_{L^{1}(0, T ; D(\mathcal{A}))}$; d'autre part: $\theta^{\prime}=\alpha^{\prime \prime}=-\mathcal{A} \alpha+g=-\beta+g$ d'où: $\theta^{\prime}(T)=-\beta(T) \in \mathcal{V}$ et

$$
\left\|\theta^{\prime}(T)\right\|_{\mathcal{V}} \leq C\|g\|_{L^{1}(0, T ; D(\mathcal{A}))}
$$

\subsection{Le problème adjoint}

On donne: $T>T_{0}, \varphi^{0}=\left(\varphi_{1}^{0}, \varphi_{2}^{0}\right) \in D(\mathcal{A}), \varphi^{1}=\left(\varphi_{1}^{1}, \varphi_{2}^{1}\right) \in \mathcal{V}$; soit $\varphi=\left(\varphi_{1}, \varphi_{2}\right)$ la solution du problème homogène (1.6); on pose:

$$
\begin{gathered}
\left\|\left(\varphi^{0}, \varphi^{1}\right)\right\|_{\mathcal{F}}^{2}=\int_{\Sigma_{T}}\left(\sigma_{T}^{0}\left(\varphi_{1}\right): \epsilon_{T}^{0}\left(\varphi_{1}\right)+\left|\varphi_{1}\right|^{2}+\left|\varphi_{1}^{\prime}\right|^{2}+\right. \\
\left.\left|\nabla_{T} \varphi_{1 \nu}\right|^{2}+\left|\sigma\left(\varphi_{1}\right) . \nu\right|^{2}\right) d \Sigma
\end{gathered}
$$

Proposition 5.2. $\|.\|_{\mathcal{F}}$ est une norme sur $D(\mathcal{A}) \times \mathcal{V}$; le complété $\mathcal{F}$ de $D(\mathcal{A}) \times \mathcal{V}$ pour cette norme est contenu dans $\mathcal{V} \times \mathcal{H}$

Preuve. D'après $(1.8)$ on a: $\left\|\left(\varphi^{0}, \varphi^{1}\right)\right\|_{\mathcal{V} \times \mathcal{H}} \leq C\left\|\left(\varphi^{0}, \varphi^{1}\right)\right\|_{\mathcal{F}}$ d'où le résultat.

Remarque 5.1. Soit $\left(\varphi^{0}, \varphi^{1}\right) \in \mathcal{F}, \varphi$ la solution du problème homogène (1.6) correspondant; alors on a $\varphi_{1}^{\prime} \in L^{2}\left(\Sigma_{T} ; \mathbb{R}^{3}\right), \partial_{\nu} \varphi_{1} \in L^{2}\left(\Sigma_{T} ; \mathbb{R}^{3}\right)$ et $\varphi_{1 \nu} \in L^{2}\left(0, T ; H^{1}(\Gamma)\right)$.

Remarque 5.2. On a:

$$
D(\mathcal{A}) \times \mathcal{V} \subset \mathcal{F} \subset \mathcal{V} \times \mathcal{H} \text { et } \mathcal{V}^{\prime} \times \mathcal{H} \subset \mathcal{F}^{\prime} \subset D(\mathcal{A})^{\prime} \times \mathcal{V}^{\prime}
$$

Proposition 5.3. Soient $f \in L^{1}(0, T ; \mathcal{V})$ et $\left\{\theta^{0}, \theta^{1}\right\} \in \mathcal{F}$. Si $\theta$ est la solution du problème:

$$
\theta^{\prime \prime}+\mathcal{A} \theta=f, \quad \theta(0)=\theta^{0}, \quad \theta^{\prime}(0)=\theta^{1}
$$

alors il existe une constante positive $C$ ne dépendant que de $\Omega$ telle que:

$$
\begin{aligned}
& \int_{\Sigma_{T}}\left(\sigma_{T}^{0}\left(\theta_{1}\right): \epsilon_{T}^{0}\left(\theta_{1}\right)+\left|\theta_{1}\right|^{2}+\left|\theta_{1}^{\prime}\right|^{2}+\left|\nabla_{T} \theta_{1 \nu}\right|^{2}+\right) \\
& \left.\left|\sigma\left(\theta_{1}\right) \cdot \nu\right|^{2}\right) d \Sigma \leq C\left(\|f\|_{L^{1}(0, T ; \mathcal{V})}+\left\|\left\{\theta^{0}, \theta^{1}\right\}\right\|_{\mathcal{F}}\right)
\end{aligned}
$$


Preuve. On pose $\theta=p+q$ où $p$ est la solution du problème avec $f=0$ et $q$ la solution avec les conditions initiales nulles. Par définition de $\mathcal{F}$ on a:

$$
\begin{gathered}
\int_{\Sigma_{T}}\left(\sigma_{T}^{0}\left(p_{1}\right): \epsilon_{T}^{0}\left(p_{1}\right)+\left|p_{1}\right|^{2}+\left|p_{1}^{\prime}\right|^{2}+\left|\nabla_{T} p_{1 \nu}\right|^{2}+\right. \\
\left.\left|\sigma\left(p_{1}\right) \cdot \nu\right|^{2}\right) d \Sigma=\left\|\left\{\theta^{0}, \theta^{1}\right\}\right\|_{\mathcal{F}}^{2}
\end{gathered}
$$

D'après le point (ii) du théorème 1.1 on a:

$$
\left(q_{1}, q_{1 T}, \sigma\left(q_{1}\right) . \nu\right) \in C^{0}\left([0, T] ; \mathbb{H}^{\frac{3}{2}}(\Omega) \times H^{2}(\Gamma, T(\Gamma)) \times \mathbb{L}^{2}(\Gamma)\right)
$$

et il existe une constante positive $C$ ne dépendant que de $\Omega$ telle que:

$$
\begin{array}{r}
\left\|q_{1}\right\|_{L^{\infty}\left(0, T ; \mathbb{H}^{\frac{3}{2}}(\Omega)\right)}+\left\|q_{1 T}\right\|_{L^{\infty}\left(0, T ; H^{2}(\Gamma, T(\Gamma))\right)}+ \\
\left\|\sigma\left(q_{1}\right) \cdot \nu\right\|_{L^{\infty}\left(0, T ; \mathbb{L}^{2}(\Gamma)\right)} \leq C\|f\|_{L^{1}(0, T ; \mathcal{V})}
\end{array}
$$

d'où

$$
\begin{gathered}
\int_{\Sigma_{T}}\left(\sigma_{T}^{0}\left(q_{1}\right): \epsilon_{T}^{0}\left(q_{1}\right)+\left|q_{1}\right|^{2}+\left|q_{1}^{\prime}\right|^{2}+\left|\nabla_{T} q_{1 \nu}\right|^{2}+\right. \\
\left.\left|\sigma\left(q_{1}\right) \cdot \nu\right|^{2}\right) d \Sigma \leq C\|f\|_{L^{1}(0, T ; \mathcal{V})} .
\end{gathered}
$$

On donne $\left(\varphi_{0}, \varphi_{1}\right) \in \mathcal{F}$ et on apelle $\varphi$ la solution du problème homogène (1.6) correspondant. Soit $\left(f,\left\{\theta^{0}, \theta^{1}\right\}\right) \in L^{1}(0, T ; \mathcal{V}) \times \mathcal{F}$; on considère la forme linéaire $L$ définie sur $\mathcal{F}$ par:

$$
\begin{gathered}
L\left(f,\left\{\theta^{0}, \theta^{1}\right\}\right)=-\int_{\Sigma_{T}}\left(\sigma_{T}^{0}\left(\varphi_{1}\right): \epsilon_{T}^{0}\left(\theta_{1}\right)+\varphi_{1} \theta_{1}+\varphi_{1}^{\prime} \theta_{1}^{\prime}+\right. \\
\left.\nabla_{T} \varphi_{1 \nu} \nabla_{T} \theta_{1 \nu}-\left(\sigma\left(\varphi_{1}\right) \nu\right)\left(\sigma\left(\theta_{1}\right) \nu\right)\right) d \Sigma
\end{gathered}
$$

où $\theta$ est la solution du problème:

$$
\theta^{\prime \prime}+\mathcal{A} \theta=f, \quad \theta(0)=\theta^{0}, \theta^{\prime}(0)=\theta^{1}
$$

Proposition 5.4. Il existe $\psi \in L^{\infty}\left(0, T ; \mathcal{V}^{\prime}\right)$ et $\left(\rho^{1},-\rho^{0}\right) \in \mathcal{F}^{\prime}$ uniques tels que:

$$
L\left(f, \theta^{0}, \theta^{1}\right)=\int_{0}^{T}\left((\psi(t), f(t)) \mathcal{V}^{\prime}, \mathcal{V}\right) d t-\left(\rho^{1}, \theta^{0}\right)+\left(\rho^{0}, \theta^{1}\right)
$$


pour tout $\left(f,\left\{\theta^{0}, \theta^{1}\right\}\right) \in L^{1}(0, T ; \mathcal{V}) \times \mathcal{F}$ et il existe une constante positive $C$ ne dépendant que de $\Omega$ telle que:

$$
\|\psi\|_{L^{\infty}\left(0, T ; \mathcal{V}^{\prime}\right)}+\left\|\left(\rho^{1},-\rho^{0}\right)\right\|_{\mathcal{F}^{\prime}} \leq C\left\|\left\{\varphi^{0}, \varphi^{1}\right\}\right\|_{\mathcal{F}}
$$

Preuve. d'après la proposition 5.3 on a:

$$
\left|L\left(f,\left\{\theta^{0}, \theta^{1}\right\}\right)\right| \leq C\left(\left\|\left\{\theta^{0}, \theta^{1}\right\}\right\|_{\mathcal{F}}+\|f\|_{L^{1}(0, T ; \mathcal{V})}\right)\left\|\left\{\varphi^{0}, \varphi^{1}\right\}\right\|_{\mathcal{F}}
$$

d'où l'existence et l'unicité de $\left(\psi,\left\{\rho^{1},-\rho^{0}\right\}\right) \in L^{\infty}\left(0, T ; \mathcal{V}^{\prime}\right) \times \mathcal{F}^{\prime}$ vérifiant (5.5).

Remarque 5.3. $\psi$ est solution formelle du problème:

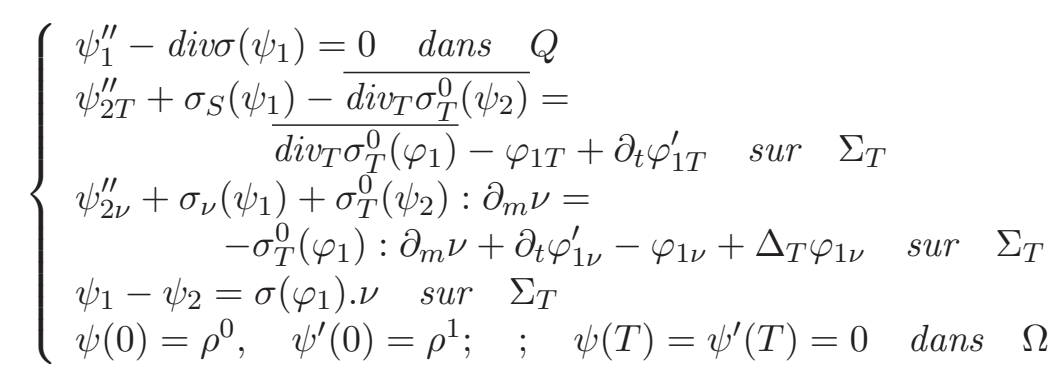

Afin de montrer que l'on peut donner un sens aux valeurs de $\psi$ et $\psi^{\prime}$ aux bornes de l'intervalle $(0, T)$ on va utiliser le résultat de régularité des solutions faibles obtenu à la proposition 5.1.

Proposition 5.5. La fonction $\psi$ définie à la proposition 5.4 est telle que:

$\psi \in \mathcal{W}^{1, \infty}\left(0, T ; D(\mathcal{A})^{\prime}\right)$, de plus $\psi(0)$ et $\psi^{\prime}(0)$ sont définis et on a: $\psi(0)=\rho^{0}, \psi^{\prime}(0)=\rho^{1} ; \psi(T)=\psi^{\prime}(T)=0$.

Preuve. On prend $f=\partial_{t} g$ dans $(5.4)$ avec $g \in L^{1}(0, T ; D(\mathcal{A}))$ et $\left\{\theta^{0}, \theta^{1}\right\}=0 ; L(f, 0,0)$ a encore un sens d'après les propositions 5.1 et 3.3 et on a:

$$
|L(f, 0,0)| \leq C_{T}\left\|\left(\varphi^{0}, \varphi^{1}\right)\right\| \mathcal{F}\|g\|_{L^{1}(0, T ; D(\mathcal{A}))}
$$

ce qui montre que $\psi \in \mathcal{W}^{1, \infty}\left(0, T ; D(\mathcal{A})^{\prime}\right)$ et donne un sens à $\psi(0)$ et $\psi(T)$ comme éléments de $D(\mathcal{A})^{\prime}$. Afin de donner un sens à $\psi^{\prime}(0)$ et $\psi^{\prime}(T)$ on procède comme dans [9]. On introduit les fonctions propres de $\mathcal{A}$ :

$$
\mathcal{A} m=\lambda m, m \in D(\mathcal{A}) .
$$


Si $f(t)=g(t) m, g \in \mathcal{D}(0, T), \theta^{0}=\alpha^{0} m, \theta^{1}=\alpha^{1} m,\left(\alpha^{0}, \alpha^{1}\right) \in \mathbb{R}^{2}$, alors la solution du problème:

$$
\theta^{\prime \prime}+\mathcal{A} \theta=f, \quad \theta(0)=\theta^{0}, \quad \theta^{\prime}(0)=\theta^{1}
$$

s'écrit: $\theta=h m$ avec:

$$
h^{\prime \prime}+\lambda h=g, \quad h(0)=\alpha^{0}, \quad h^{\prime}(0)=\alpha^{1}
$$

en posant:

$$
\begin{gathered}
\xi_{0}(t)=-\int_{\Gamma} \varphi_{1}^{\prime} m_{1} d \Gamma, \quad \text { et } \\
\xi_{1}(t)=-\int_{\Gamma}\left(\sigma_{T}^{0}\left(\varphi_{1}\right): \epsilon_{T}^{0}\left(m_{1}\right)+\varphi_{1} m_{1}+\nabla_{T} \varphi_{1 \nu} \nabla_{T} m_{1 \nu}\right) d \Gamma- \\
\int_{\Gamma}\left(\sigma\left(\varphi_{1}\right) \cdot \nu\right)\left(\sigma\left(m_{1}\right) \cdot \nu\right) d \Sigma
\end{gathered}
$$

on obtient:

$$
\int_{0}^{T}(\psi, m)\left(h^{\prime \prime}+\lambda h\right) d t+\alpha_{1}\left(\rho^{0}, m\right)-\alpha_{0}\left(\rho^{1}, m\right)=\int_{0}^{T}\left(\xi_{0} h^{\prime}+\xi_{1} h\right) d t .
$$

Comme $h, \alpha_{0}$ et $\alpha_{1}$ sont arbitraires, $(\psi, m)$ vérifie:

$$
(\psi, m)^{\prime \prime}+\lambda(\psi, m)=\xi_{1}-\partial_{t} \xi_{0}
$$

avec: $\xi_{0}, \xi_{1} \in L^{2}(0, T)$; cela donne un sens à $(\psi, m)^{\prime}(0)$ et $(\psi, m)^{\prime}(T)$ $(c f .[9])$, et on a:

$$
\begin{gathered}
(\psi, m)(T) h^{\prime}(T)-(\psi, m)^{\prime}(T) h(T)+(\psi, m)^{\prime}(0) h(0)- \\
(\psi, m)(0) h^{\prime}(0)=h(0)\left(\rho^{1}, m\right)-h^{\prime}(0)\left(\rho^{0}, m\right)
\end{gathered}
$$

comme h est arbitraire, on obtient les valeurs annoncées.

Remarque 5.4. Précisons le sens des conditions vérifiées par $\psi$.

- $\overline{\operatorname{div}_{T} \sigma_{T}^{0}\left(\varphi_{2}\right)}$ est un élément de $L^{2}\left(0, T: H^{-1}(\Gamma, T(\Gamma))\right.$ défini par:

$$
\left(\overline{d i v_{T} \sigma_{T}^{0}\left(\varphi_{2}\right)}, v_{T}\right)=-\int_{\Sigma_{T}} \sigma_{T}^{0}\left(\varphi_{2}\right): \pi\left(\partial_{m} v_{T}\right) \pi d \Sigma
$$

pour tout élément $v_{T} \in L^{2}\left(0, T: H^{1}(\Gamma, T(\Gamma))\right.$.

- $\partial_{t} \varphi_{1 T}^{\prime}$ est un élément de $\left(H^{1}\left(0, T ; L^{2}(\Gamma, T(\Gamma))\right)\right)^{\prime}$ défini par:

$$
\left(\partial_{t} \varphi_{1 T}^{\prime}, v_{T}\right)=-\int_{\Sigma_{T}} \overline{\varphi_{1 T}^{\prime}} v_{T}^{\prime} d \Sigma, \forall v_{T} \in H^{1}\left(0, T ; L^{2}(\Gamma, T(\Gamma))\right) .
$$


$\circ \Delta_{T \varphi_{1} \nu \mid \Gamma}$ est un élément de $L^{2}\left(0, T ; H^{-1}(\Gamma)\right)$ défini par:

$$
\begin{gathered}
\left(\Delta_{T} \varphi_{1 \nu}, v\right)=-\int_{\Sigma_{T}} \nabla_{T} \varphi_{1 \nu} \nabla_{T} v d \Sigma, \forall v \in L^{2}\left(0, T ; H^{1}(\Gamma)\right), \\
\left(H_{0}^{1}(\Gamma)=H^{1}(\Gamma)\right)
\end{gathered}
$$

\subsection{Le contrôle}

Fin de la démonstration du théorème 1.3.

Soit: $\Lambda: \mathcal{F} \longmapsto \mathcal{F}^{\prime}, \quad\left(\varphi^{0}, \varphi^{1}\right) \longmapsto\left(\psi^{\prime}(0),-\psi(0)\right)$ où $\psi$ est définie par (5.4). L'opérateur $\Lambda$ est un isomorphisme de $\mathcal{F} \longmapsto \mathcal{F}^{\prime}$; en effet:

$$
\begin{aligned}
& \left\langle\Lambda\left\{\varphi^{0}, \varphi^{1}\right\},\left\{\varphi^{0}, \varphi^{1}\right\}\right\rangle_{\mathcal{F}^{\prime}, \mathcal{F}}=\left(\psi^{\prime}(0), \varphi^{0}\right)- \\
& \left(\psi(0), \varphi^{1}\right)=-L\left(0, \varphi^{0}, \varphi^{1}\right)=\left\|\left\{\varphi^{0}, \varphi^{1}\right\}\right\|_{\mathcal{F}}^{2}
\end{aligned}
$$

On conclut grâce à la symétrie de $\Lambda$.

Soit $\left\{\rho^{1},-\rho^{0}\right\} \in \mathcal{F}^{\prime}$ et $\left(\varphi^{0}, \varphi^{1}\right)=\Lambda^{-1}\left\{\rho^{1},-\rho^{0}\right\}$; soit $\varphi$ la solution $\mathrm{du}$ problème homogène (1.6) avec les conditions initiales $\left(\varphi^{0}, \varphi^{1}\right)$. Il suffit de prendre $\mathbf{u}=\psi$ où $\psi$ est définie par (5.4); le controle $\mathbf{g}$ est donné par la remarque 5.3 .

\section{References}

[1] Destuynder, Ph. Modélisation des coques minces élastiques, Cours École Centrale des Arts et Manufactures.

[2] G. Duvaut et J.L. Lions, Les inéquations en mécanique et en physique, Dunod, Paris (1972).

[3] A. Heminna, Contrôlabilité exacte d'un problème avec conditions de Ventcel évolutives..., C.R.Acad. Sci. Paris, 324, série I (1997) 195-200.

[4] A. Heminna, Stabilisation de problèmes de Ventcel, C.R.Acad. Sci. Paris, 328, série I (1999) 1171-1174.

[5] V. Komornik, 1994. Exact controllability and stabilization; the multiplier method, Masson-John Wiley, Paris.

[6] T.J.R. Hughes and J.E. Marsden, Mathematical foundations of elasticity, Prentice-Hall, Inc., Englewood Cliffs, New Jersey 07632.

[7] K. Lemrabet, Etude de divers problèmes aux limites de Ventcel d'origine physique ou mécanique dans des domaines non réguliers, Thèse, U S T H B, Alger (1987). 
[8] K. Lemrabet, Problème de Ventcel pour le système de l'élasticité dans un domaine de $\mathbb{R}^{3}$, C.R.Acad. Sci. Paris, 304, série I (1987) 151-154.

[9] J.L. Lions, Contrôlabilité exacte, perturbation et stabilisation de systèmes distribués, 1, Masson (1986).

[10] J.L. Lions et E. Magenes, Problèmes aux limites non homogènes et leurs applications, 1, Dunod, Paris (1968).

[11] E. Sanchez-Palencia, D. Leguillon, Computations of singular problems and elasticity, R.M.A., Masson, Paris (1987).

[12] R. Valid, La mécanique des milieux continus et le calcul des structures, Eyrolles, Paris (1977).

Amar Heminna

Institut de Mathématiques, USTHB

B.P. 32, EL-Alia, 16111

Alger, Algérie

E-mail: hemina@usa.net
Recibido: 27 de Abril de 1999

Revisado: 10 de Mayo de 2000 